\title{
miR-302a-5p/367-3p-HMGA2 axis regulates malignant processes during endometrial cancer development
}

\author{
Jian Ma, Da Li, Fan-Fei Kong, Di Yang, Hui Yang and Xiao-Xin Ma*
}

\begin{abstract}
Background: Metastasis is one of the main reasons for treatment failure in endometrial cancer. Notably, high mobility group AT-hook 2 (HMGA2) has been recognized as a driving factor of tumour metastasis. microRNAs (miRNAs) are powerful posttranscriptional regulators of HMGA2.
\end{abstract}

Methods: The binding sites of miR-302a-5p and miR-367-3p on HMGA2 mRNA were identified using bioinformatics prediction software and were validated via luciferase assay. The expression levels of miR-302a-5p and miR-367-3p were detected using quantitative real-time PCR and in situ hybridization. Western blotting and immunohistochemistry were used to detect the levels of HMGA2 and epithelial-mesenchymal transition pathwayrelated proteins. Co-immunoprecipitation was used to detect protein interactions. The roles of miR-302a-5p and miR-367-3p in the regulation of HMGA2 during the progression of endometrial cancer were investigated using both in vitro and in vivo assays.

Results: In the present study, high HMGA2 expression was correlated with poor clinical outcomes in endometrial cancer. The binding sites of miRNAs on HMGA2 mRNA were identified using bioinformatics prediction software and were validated via luciferase assay. In the endometrial cancer cell lines Ishikawa and HEC-1A, the overexpression of miR-302a-5p/367-3p significantly inhibited the expression of HMGA2 mRNA. In endometrial cancer tissues, we showed that miR-302a-5p and miR-367-3p were significantly downregulated and thus inversely correlated with HMGA2. The miR-302a-5p and miR-367-3p expression levels were closely correlated with FIGO stage and lymph node metastasis. High expression of miR-302a-5p/367-3p was correlated with high survival rates in endometrial cancer. In addition, miR-302a-5p/367-3p suppressed the malignant behaviour of endometrial carcinoma cells via the inhibition of HMGA2 expression.

Conclusion: Our findings indicate that miR-302a-5p/367-3p-mediated expression of HMGA2 regulates the malignant behaviour of endometrial carcinoma cells, which suggests that the miR-302a-5p/367-3p-HMGA2 axis may be a predictive biomarker of endometrial cancer metastasis and patient survival and a potential therapeutic target in metastatic endometrial cancer.

Keywords: miRNAs, HMGA2, Regulation, EMT, Endometrial cancer

\footnotetext{
* Correspondence: maxiaoxin666@aliyun.com

Department of Obstetrics and Gynecology, Key Laboratory of Maternal-Fetal

Medicine of Liaoning Province, Key Laboratory of Obstetrics and Gynecology

of Higher Education of Liaoning Province, Shengjing Hospital of China

Medical University, Shenyang 110004, China
}

(c) The Author(s). 2018 Open Access This article is distributed under the terms of the Creative Commons Attribution 4.0 International License (http://creativecommons.org/licenses/by/4.0/), which permits unrestricted use, distribution, and reproduction in any medium, provided you give appropriate credit to the original author(s) and the source, provide a link to the Creative Commons license, and indicate if changes were made. The Creative Commons Public Domain Dedication waiver (http://creativecommons.org/publicdomain/zero/1.0/) applies to the data made available in this article, unless otherwise stated. 


\section{Background}

With increasing incidence over the years, endometrial cancer is the most common malignancy of the female reproductive tract in developed countries [1]. Treatment of endometrial cancer includes surgery supplemented with radiotherapy, chemotherapy, and therapy with hormones or targeted therapy such as rapamycin [2]. For patients with advanced metastatic or recurrent endometrial cancer, treatment failure is still high due to the loss of opportunity for surgery. With the elucidation of molecular mechanisms in endometrial cancer, an increasing number of molecular targeted drugs are being tested for clinical application, and some have shown beneficial effects $[3,4]$. Therefore, the potential mechanisms in the development of endometrial cancer, especially those involved in tumour metastasis, have become the basis for the development of molecular targeted therapy for endometrial cancer, which is essential for the treatment of this type of cancer.

High mobility group AT-hook 2 (HMGA2) is a chromatin-binding protein that organizes protein complexes on enhancers of various genes to regulate gene expression and cell differentiation [5]. HMGA2 has emerged as a candidate tumour biomarker [6] because it is overexpressed in many cancer patients and is undetectable in adults under normal physiological conditions [7]. In addition, the expression of the HMGA2 is associated with tumour stage and clinical outcomes in ovarian carcinoma [8]. As an oncogene, HMGA2 regulates cell proliferation, the cell cycle and apoptosis, and it is involved in the regulation of invasion and metastasis, which are key processes in tumour progression, such as ovarian tumours [9], breast adenocarcinoma [10] and lung cancers [11]. Until now, it has been reported that HMGA2 is highly expressed in endometrial cancer tissues and that the overexpression of HMGA2 promotes tumour growth and metastasis [12]. However, the mechanisms by which HMGA2 regulates the biological characteristics of endometrial cancer as well as the factors involved remain undefined.

MicroRNAs (miRNAs) are molecules that can regulate a set of target genes and lead to translational repression or mRNA degradation. Recent studies have demonstrated extensive deregulation of miRNAs in endometrial carcinoma, which regulate cell growth and metastasis and affect tumour initiation and development [13]. miRNAs regulating the activity of HMGA2 are considered major molecular mediators in tumour metastasis [14].

The epithelial-mesenchymal transition (EMT) is a central process in the invasion and metastasis of human tumours $[15,16]$. HMGA2 is a driving factor of this process [17-19]. Identification of the mechanisms triggering EMT may serve as an efficient strategy for the treatment of malignant and metastatic tumours.
In this study, we identified two novel aberrantlyexpressed miRNAs, miR-302a-5p and miR-367-3p, that modulate the malignant behaviour of endometrial carcinoma cells through the suppression of HMGA2 expression.

\section{Methods \\ Bioinformatics prediction}

To predict miRNAs with potential binding sites on HMGA2 mRNA, we employed 2 types of bioinformatics software, namely, TargetScan 6.0 (http://www.targetsca n.org/) and miRanda (http://www.microrna.org/microrna/ home.do). To analyse the clinicopathologic parameters and overall survival curves of the endometrial carcinoma patients with high or low HMGA2 mRNA expression and the relationship between HMGA2 protein and RUNX1 protein levels, data from The Cancer Genome Atlas (TCGA) dataset were retrieved and analysed.

\section{Patients and samples}

Forty endometrial cancer tissues, 37 normal fresh endometrial tissues and 99 cases of paraffin-embedded tissue sections (80 endometrial cancers and 19 normal endometrial tissues) were obtained from the Department of Obstetrics and Gynaecology, Shengjing Hospital of China Medical University from patients undergoing surgical resection from 2011 to 2017, excluding patients with liver and kidney disease and patients without preoperative radiotherapy and chemotherapy. Postoperative histopathology of paraffin sections was used to confirm the diagnosis of endometrial cancer according to the International Union of Obstetrics and Gynaecology (FIGO 2009) staging standards. Samples from normal intimal lining of the uterus at proliferative and secretory phases were used as controls. All protocols were approved by the agency, and consent was received from the patients.

\section{Cell culture}

The endometrial cancer cell lines Ishikawa and HEC-1A were maintained in RPMI 1640 medium (Gibco, Carlsbad, CA, USA) and McCoy's 5A medium (Gibco) respectively. The human embryonic kidney cell line HEK293T was maintained in high-glucose DMEM (Gibco). The cells were cultured in the presence of $10 \%$ foetal bovine serum (FBS) (Gibco), $50 \mathrm{IU} / \mathrm{mL}$ penicillin, and $50 \mathrm{mg} / \mathrm{mL}$ streptomycin (Invitrogen, Carlsbad, CA, USA). All cells were cultured at $37{ }^{\circ} \mathrm{C}$ in a humidified incubator in the presence of $5 \% \mathrm{CO}_{2}$. All cells were obtained from the Institute of Biochemistry and Cell Biology, Chinese Academy of Sciences (Shanghai, China).

\section{Transfection of cells}

The overexpression plasmid (gV144-HMGA2), knockdown plasmid (sh-HMGA2) and their respective negative control $(\mathrm{NC})$ counterparts were purchased from 
GeneChem (Shanghai, China). Agomir, antagomir and scrambled negative control RNAs were purchased from GenePharma (Shanghai, China). Lipofectamine 3000 (Invitrogen) was used to transfect cells with miRNAs and HMGA2 for subsequent experiments according to the manufacturer's instructions. The sequences of the shRNA clone and the agomir and antagomir are listed in Additional file 1: Table S1.

\section{RNA extraction and quantitative RT-PCR}

RNA was extracted from tissue or cells with TRIzol (Takara, Dalian, China). After measurement of the RNA concentration, cDNAs were generated from reverse transcription of RNA. cDNAs corresponding to the mRNAs of interest were reverse transcribed using PrimeScript RT-polymerase (Takara). cDNAs corresponding to the miRNAs of interest were synthesized using Mir-X miRNA First-Strand Synthesis kit (Clontech, Dalian, China), and SYBR Green Premix (Takara) was used to perform quantitative real-time PCR (qRT-PCR) according to the manufacturer's instructions. The specific PCR primers were designed by Sangon Biotech Co. Ltd. (Shanghai, China). With GAPDH and U6 as internal controls, fold-changes were calculated using the $2^{-\Delta \Delta C t}$ method. The primer sequences are listed in Additional file 2: Table S2.

\section{Protein extraction, western blotting and co-immunoprecipitation assay}

Total protein was extracted with the Protein Extraction kit (Beyotime Biotechnology, Shanghai, China). Proteins were separated using $10 \%$ sodium dodecyl sulphate (SDS)-polyacrylamide gel electrophoresis (PAGE) and then transferred to polyvinylidene difluoride (PVDF) membranes (Millipore, MA, USA). Then, the membranes were incubated with primary antibodies overnight at $4{ }^{\circ} \mathrm{C}$ (the primary antibodies are listed in Additional file 3: Table S3) and subsequently incubated with secondary antibodies for $1.5 \mathrm{~h}$ at room temperature. After being washed, the membranes were visualized using Quantity One imaging software (Bio-Rad, CA, USA). For coimmunoprecipitation experiments, ice-cold RIPA buffer was added to the cells. After centrifugation, the supernatant fractions were collected and treated with an antiHMGA2 antibody for $3 \mathrm{~h}$ at $4{ }^{\circ} \mathrm{C}$. Protein A/G PLUSAgarose (Santa Cruz Biotechnology, Santa Cruz, CA, USA) was then added, followed by overnight incubation at $4{ }^{\circ} \mathrm{C}$ on a rocker platform. Then, the samples were processed following the protocols for western blotting. The intensities of the protein bands were quantified using ImageJ software v.1.48 [http://imagej.nih.gov/ij/ download.html].

\section{Enzyme-linked immunosorbent assay}

The levels of matrix metalloproteinase-2 (MMP-2) and matrix metalloproteinase-9 (MMP-9) in the conditioned medium were quantified using Quantikine ELISA kits (R\&D systems, Minneapolis, USA) per the manufacturer's guidelines.

\section{Luciferase assay}

HEK293T cells were plated in 96-well plates. The cells were co-transfected with miRNAs or scrambled NC (RiboBio, Guangzhou, China) and $100 \mathrm{ng}$ of wild-type or mutant dual-luciferase reporter vector containing an HMGA2 gene fragment (pmiR-RB-Report-HMGA2) (RiboBio) using Lipofectamine 3000. After $48 \mathrm{~h}$ of cell transfection, the luciferase activity was measured using the Dual-Luciferase Reporter Assay System (Promega, Madison, WI, USA). The experiments were performed in triplicate.

\section{Cell proliferation assay}

Cells were seeded in 96-well plates, and $10 \mu \mathrm{L}$ CCK-8 (Cell Counting Kit-8; Dojindo, Japan) reagent was added per well. After incubation for $3 \mathrm{~h}$ at $37{ }^{\circ} \mathrm{C}$ in $5 \% \mathrm{CO}_{2}$, the OD at $450 \mathrm{~nm}$ of each well was measured with a microplate reader at $24 \mathrm{~h}, 48 \mathrm{~h}$ and $72 \mathrm{~h}$. We detected cell proliferation with an EdU Cell Proliferation Assay kit (RiboBio). Cells were incubated with $50 \mu \mathrm{M}$ EdU for $2 \mathrm{~h}$, and then to stain the DNA, $100 \mu \mathrm{L}$ of $1 \mu \mathrm{g} / \mathrm{mL}$ Hoechst 3342 reaction mixture was added to each well for $30 \mathrm{~min}$. The cells were imaged using a fluorescence microscope (Nikon, Japan). Original magnification: $\times 20$. The experiments were performed in triplicate.

\section{Wound healing assay}

Cells were grown to approximately 90\% confluency in 6well plates, and a straight wound was artificially created on the cell monolayer with a $100-\mu \mathrm{L}$ pipette tip. The cells were washed with serum-free medium. The ability of cells to migrate to the site of the wound was observed under the microscope at daily intervals, and photographs were captured at $0 \mathrm{~h}$ and $48 \mathrm{~h}$. The experiments were performed in triplicate. Original magnification: $\times 10$.

\section{Cell invasion assay}

The transwell method was used to detect cell invasion. Transwell filter inserts $(8-\mu \mathrm{M}$ pore size; Corning, NY, USA) were pre-coated with Matrigel for $30 \mathrm{~min}$ at $37^{\circ} \mathrm{C}$ and washed with serum-free medium. Cells were suspended in $200 \mu \mathrm{L}$ serum-free medium and placed in the upper chambers, and the lower chambers were filled with $700 \mu \mathrm{L}$ of culture medium containing $10 \%$ FBS as the chemo attractant. After several hours, the noninvading cells on the upper surface of the membranes were wiped off with cotton swabs, and $4 \%$ 
paraformaldehyde was used to fix the invading cells for 50 min, which was followed by staining with $0.1 \%$ crystal violet and washing. The invaded cells were quantified via imaging under an inverted fluorescence microscope equipped with an image acquisition system (Nikon, Japan). The experiments were performed in triplicate. Original magnification: $\times 20$.

\section{Apoptosis and cell cycle analyses}

Cellular apoptosis was detected via flow cytometry. Cells were transfected for $48 \mathrm{~h}$, and $10^{6}$ cells were harvested per group. The cells were washed and stained using an apoptosis detection kit for double staining with Annexin VAPC/7-AAD (KeyGen Biotech, Nanjing, China). After incubation for $15 \mathrm{~min}$, the cells were analysed using flow cytometry (BD FACSCalibur, NJ, USA). Cell cycle distribution was analysed via flow cytometry. After transfection, the cells were harvested, washed and fixed with $70 \%$ ethanol at $4{ }^{\circ} \mathrm{C}$ overnight. The cells were mixed with $100 \mu \mathrm{L}$ of RNase, and cellular DNA was stained using PI. The cells were analysed via flow cytometry (BD.

FACSCalibur), and the results were compared with those of cell proliferation assays. The experiment was performed in triplicate.

\section{Tumour xenografts in nude mice}

Tumourigenesis experiments in nude mice were performed in strict accordance with the protocol approved by the Scientific Research and New Technology Ethical Committee of the Shengjing Hospital of China Medical University. Four- to six-week-old (BALB/c) athymic nude mice were purchased from HFK Bioscience Co., Ltd. (Beijing, China). Cells were harvested, suspended at $10^{6}$ cells $/ 100 \mu \mathrm{L}$ of PBS and subcutaneously injected into the left axilla of the mice. For treatment, the respective agents were injected into tumours at multiple sites every 4 days. Tumour volume was measured every 4 days, and the mice were sacrificed after 28 days. The excised tumours were fixed at $4{ }^{\circ} \mathrm{C}$ in paraformaldehyde and embedded in paraffin. Tumour sections were used for immunohistochemistry analyses. Each group contained three mice.

\section{Immunohistochemistry and in situ hybridization}

Subcutaneous tumours were excised, fixed in $4 \%$ paraformaldehyde, dehydrated, embedded in paraffin and sectioned for immunohistochemical analyses. For in situ hybridization (ISH), the digoxigenin-labeled oligonucleotide miR-302a-5p, miR-367-3p detection probe (Boster Biological Technology Co., Ltd., Nanjing, China) was used, and the miR-302a-5p probe sequence was as follows: $\left(5^{\prime}-3^{\prime}\right)$ AGCAAGTACATCCACGTTTAAGT, miR-367-3p (5'-3') TCACCATTGCTAAGTGCAATT. The experiments were performed according to the in situ hybridization test kit instructions. The immunostained sections were observed under an optical microscope and scored according to the percentage of positive cells, as follows: less than $10 \%$ positive cells, $1 ; 10 \%$ to $50 \%$ positive cells, 2 ; and 51 to $75 \%$ positive cells, 3 . The staining intensity was scored as weak, 1 ; medium, 2 ; and strong, 3. The final score was calculated by multiplying the score for staining intensity with the score corresponding to the percentage of positive cells. Thus, a total score of 1 to 9 was calculated, as follows: $\leq 2$ points as negative, $3 \sim 4$ as weak positive $(+), 5 \sim 8$ as moderate positive $(++)$, and 9 as strong positive $(+++) .-1+$ indicated low expression, $++1+++$ indicated high expression. Original magnification: $\times 400$.

\section{Statistical analysis}

The data were presented as the mean \pm SEM of 3 independent experiments. All statistical analyses were performed with GraphPad Prism 6.0 (La Jolla, CA) and SPSS 17.0 software (Abbott Laboratories, Chicago, IL). Student's $t$ test and the Chi-square test were used for comparisons between two independent groups, and oneway ANOVA was used to compare differences among more than two groups. Pearson's and Spearman's correlation coefficient analyses were used for the determination of correlation. Survival curves were plotted according to the Kaplan-Meier method and were compared using the log-rank test. Disease-free survival was defined as the time to progression or last follow-up or death from the date of diagnosis. $P<0.05$ was defined as statistically significant; $P>0.05$ indicated non-significance (NS).

\section{Results}

High HMGA2 expression correlates with poor clinical outcomes in endometrial cancer

The results of qRT-PCR and western blotting showed that the expression level of HMGA2 was higher in endometrial carcinoma tissues than that in normal endometrial tissues (Fig. 1a and b). The immunohistochemistry results showed that the positive rate of HMGA2 protein expression in endometrial cancer was $80.0 \%$, which was much higher than that of normal endometrial tissue (10.5\%) (Fig. 1d, Additional file 4: Table S4-i). In addition, we analysed the association of the levels of HMGA2 protein (Additional file 4: Table S4-ii) and mRNA (Additional file 5: Table S5) with the clinicopathologic parameters and the disease-free survival of endometrial cancer patients. The results showed that HMGA2 expression was significantly associated with the clinicopathological features. HMGA2 was increased in the progression from stage I to stages III \& IV. In addition, we found that HMGA2 expression was significantly associated with tumour grade and myometrial invasion in patients with endometrial cancer and that 


\section{a}
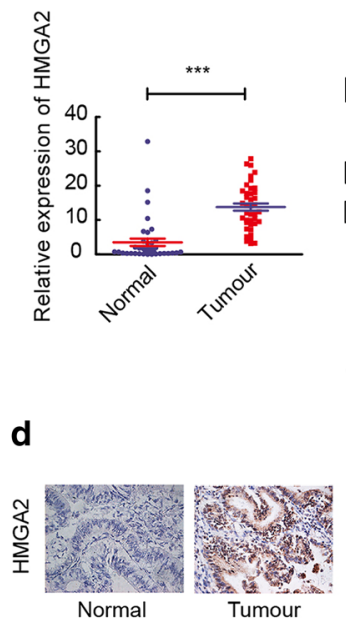

g

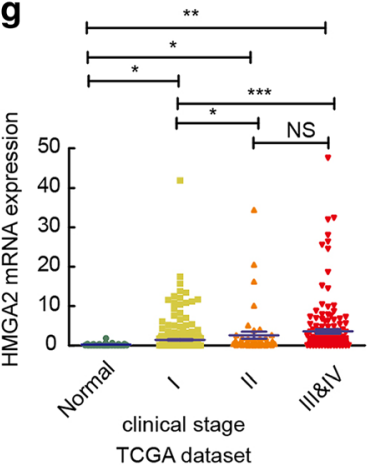

j

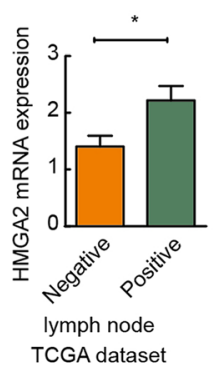

T1 T2 N1 N2

I

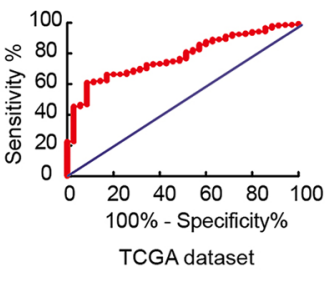

- Low expression of HMGA2 protein - High expression of HMGA2 protein

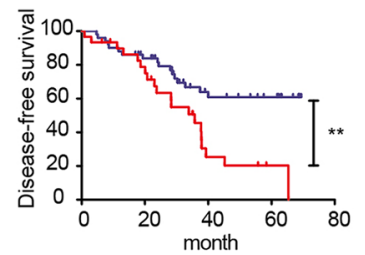

C

- Low expression of HMGA2 mRNA - High expression of HMGA2 mRNA

b-i
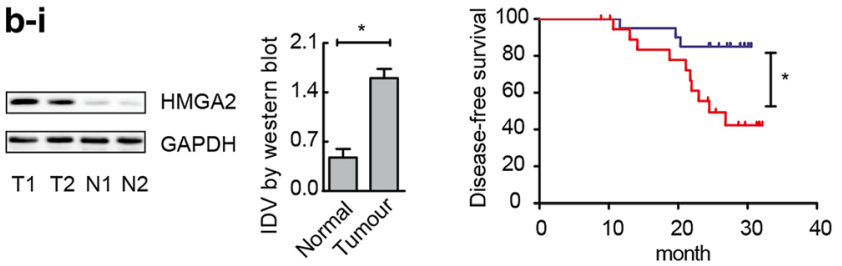

$\mathbf{f}$

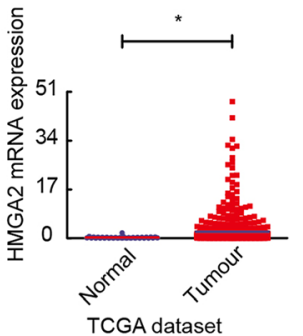

i
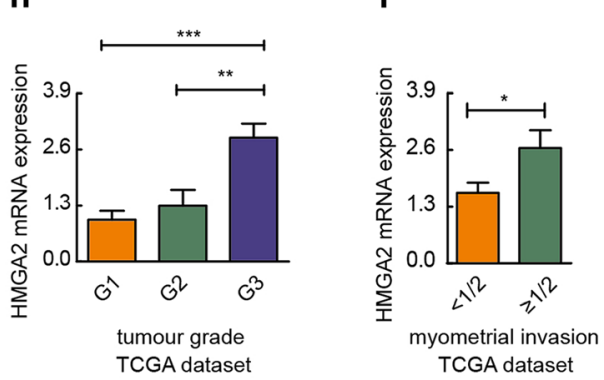

- Low expression HMGA2 mRNA

- High expression HMGA2 mRNA

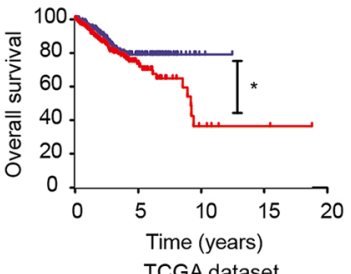

Fig. 1 High expression of HMGA2 correlates with worse clinical outcomes in endometrial cancer patients. $\mathbf{a}$ and $\mathbf{b}$ HMGA2 was up-regulated in endometrial carcinoma tissues $(n=40)$ compared with normal tissues $(n=37)$. Data are presented as the mean \pm SEM. c Disease-free survival curves for HMGA2 mRNA in 40 endometrial carcinoma cases. d The expression of HMGA2 was detected via immunohistochemistry in endometrial cancer $(n=80)$ and normal endometrial tissue $(n=19)$. e Disease-free survival curves for HMGA2 protein in 80 endometrial carcinoma cases.

f Compared with normal endometrial tissue $(n=35)$, (the controls were collected from paracancerous tissues in patients with endometrial cancer). HMGA2 was highly expressed in 552 endometrial carcinoma samples (TCGA cohort). g HMGA2 expression levels in patients with different clinical stages of endometrial cancer (TCGA cohort): normal $(n=35)$, I $(n=339)$, II $(n=48)$, III \& IV $(n=153)$. h HMGA2 expression levels in patients with endometrial cancers of different grades (TCGA cohort): G1 $(n=109), \mathrm{G} 2(n=120), \mathrm{G} 3(n=314)$. i Myometrial invasion (TCGA cohort): < $1 / 2$ group ( $n=259), \geq 1 / 2$ group $(n=211)$. j Lymph node status (TCGA cohort): negative group $(n=190)$, positive group $(n=322)$. $\mathbf{k}$ ROC of HMGA2 (TCGA cohort). I High expression of HMGA2 predicted a shorter overall survival in endometrial cancer. The data were retrieved and analysed from the TCGA dataset $(n=542) .{ }^{*} P<0.05,{ }^{*} P<0.01,{ }^{* *} P<0.0001$ 
HMGA2 expression levels were significantly upregulated in the tissues of endometrial cancer patients with lymph node metastasis compared with those of patients without lymph node metastasis. The disease-free survival curves for the endometrial carcinoma patients with high or low HMGA2 mRNA (Fig. 1c) and protein expression (Fig. 1e) indicated that high expression of HMGA2 correlates with poor clinical outcomes in endometrial cancer. Based on the TCGA dataset, HMGA2 showed a dramatic overexpression in endometrial cancer tissue compared with that in normal endometrial tissues (Fig. 1f). Moreover, based on the TCGA cohort, we analysed the association between the levels of HMGA2 and the clinicopathologic parameters of endometrial cancer patients. We found that HMGA2 expression was significantly associated with clinical stage, differentiation, infiltration depth and lymphatic metastasis (Fig. 1g-j). Then, we examined the sensitivity and specificity of HMGA2. A receiver operating characteristic (ROC) curve analysis was performed, and the correlation area under the curve (AUC) was used to confirm the diagnostic efficacy of HMGA2. As shown in Fig. 1k, the AUC of HMGA2 reached 0.7761 , and the cut-off value was 0.4121 , (95\% CI: 0.7140 - 0.8382). These results suggest that HMGA2 can discriminate between endometrial carcinoma and normal endometrial tissue. To analyse the overall survival curves for the endometrial carcinoma patients in reference to HMGA2 mRNA expression, we retrieved and analysed the data from the TCGA dataset. The results showed that high expression of HMGA2 correlates with a lower survival rate in endometrial cancer (Fig. 11).

\section{Knockdown of HMGA2 inhibits the malignant behaviours of Ishikawa and HEC-1A cell lines}

In order to investigate the carcinogenic effect of HMGA2 in endometrial carcinoma, we determined the baseline expression of HMGA2 in four endometrial cancer cell lines by qRT-PCR (Additional file 6: Figure S1a). HEC-1A cells exhibited the lowest expression, while Ishikawa cells exhibited the highest expression. We therefore transfected the HMGA2 plasmid into these two cell lines. qRT-PCR and western blot analyses were used to determine the transfection efficiency of HMGA2 (Additional file 6: Figure S1b and c). The effect of HMGA2 on the proliferation of the endometrial cancer cell lines Ishikawa and HEC-1 was studied using CCK-8 and EdU assays after overexpressing or knocking down HMGA2. The proliferation of cells in the sh-HMGA2transfected group was lower than that in the sh-NCtransfected group. Additionally, the proliferation of cells in the HMGA2-overexpression group was significantly higher than that in the control group (Fig. 2a and b). Furthermore, the ability of the cells to migrate and invade was investigated, and this ability was lower in the
sh-HMGA2-transfected group than that in the sh-NC group (Fig. 2c and d). Flow cytometry was used to detect apoptosis. The rate of apoptosis in the sh-HMGA2transfected group was higher than that in the sh-NC group, and it was reduced in response to the overexpression of HMGA2 (Fig. 2e). Cell cycle distribution was analysed using flow cytometry. HMGA2 knockdown induced cell cycle arrest in the G0/G1 phase (Fig. 2f). Thus, knockdown of HMGA2 exerts tumour-suppressive effects on Ishikawa and HEC-1A cells, while overexpression of HMGA2 promotes the malignant behaviours of these cells, indicating the role of HMGA2 as an oncogene in endometrial cancer.

\section{The expression of miR-302a-5p/367-3p is low in endometrial carcinoma tissues, and HMGA2 is a target of miR-302a-5p and miR-367-3p}

TargetScan and miRanda bioinformatics databases were used to predict miRNAs that can bind to HMGA2. In total, 25 miRNAs were identified, which were screened via co-transfection of HEK293T cells with wild-type dualluciferase vector-mediated HMGA2 constructs. A significant decrease in luciferase activity was observed in response to transfection with miR-302a-5p, miR-1297, miR-367-3p, miR-365a-3p and miR-9-5p (Additional file 7: Figure S2). Furthermore, when miR-302a-5p, miR-367-3p, miR-365a-3p, miR-9-5p and miR-1297 were overexpressed in Ishikawa and HEC-1A cells, the expression level of HMGA2 was decreased most obviously after miR-302a$5 p / 367-3 p$ overexpression. Conversely, when cells were transfected with the antagomir-302a-5p/367-3p, HMGA2 expression significantly increased (Fig. 3a), which indicates that HMGA2 expression is likely regulated by miR-302a5p/miR-367-3p. qRT-PCR was used to determine the transfection efficiency (Additional file 8: Figure S3). In tissues derived from 40 cases of endometrial cancer, the results of qRT-PCR showed that the expression of miR302a-5p/367-3p in endometrial carcinoma was lower than that in the normal endometrium (Fig. 3b). Moreover, the expression of miR-302a-5p/367-3p was negatively correlated with the expression of HMGA2 (Pearson's rank correlation method; miR-302a-5p $\left(r^{2}=0.3888, P<0.0001\right)$ and miR-367-3p $\left(\mathrm{r}^{2}=0.2961, P=0.0003\right)$ (Fig. 3c). In addition, we analysed the association of the levels of miR302a-5p/367-3p with the clinicopathologic parameters (Additional file 9: Table S6; Additional file 10: Table S7) and the disease-free survival (Fig. 3d) of endometrial cancer patients. Furthermore, the expression of miR-302a-5p and miR-367-3p in 80 cases of endometrial carcinoma and in 19 normal endometrial tissues was detected by ISH assay. The results showed that the positive rate of miR302a-5p expression in endometrial cancer was $43.75 \%$, which was much lower than that in normal endometrial tissue (84.2\%) (Fig. 3e, Additional file 11: Table S8-i); the 
a-i

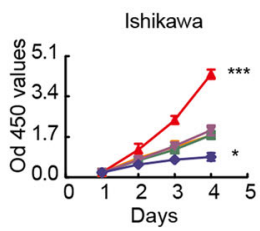

a-ii

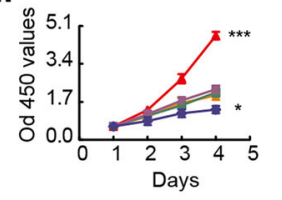

- Control

- gV144-NC

+ gV-144-HMGA2

$\neq$ sh-NC

sh-HMGA2

b-i

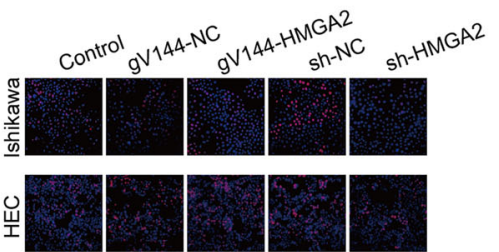

b-ii

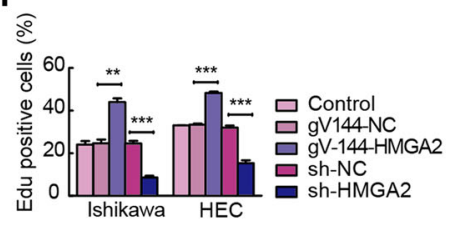

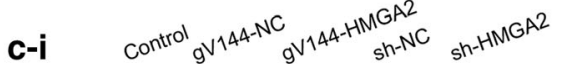
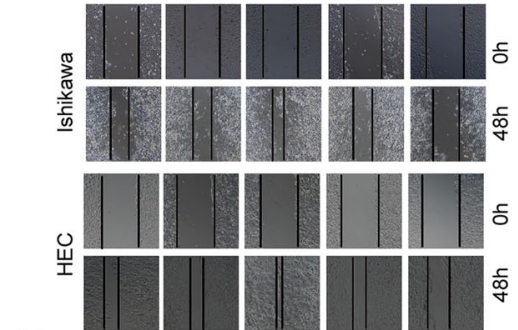

d-i

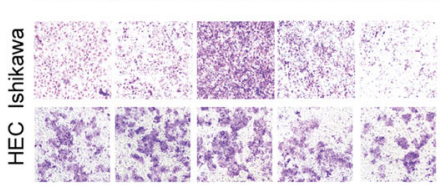

c-ii

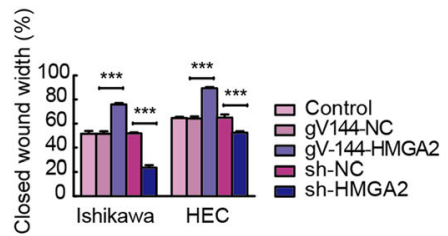

交 $\mathbf{d}-\mathbf{i i}$

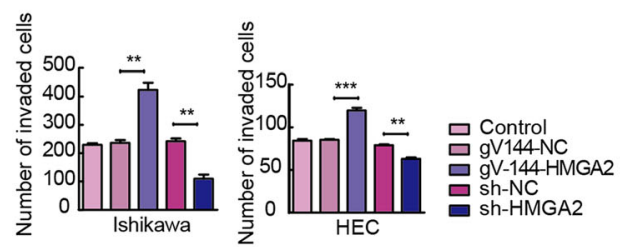

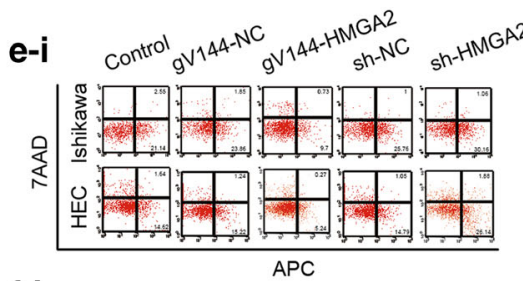

f-i
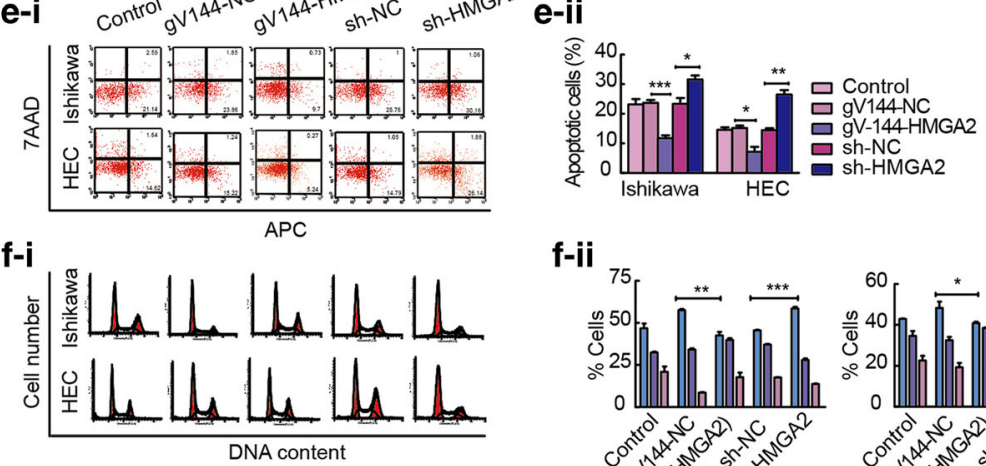

f-ii

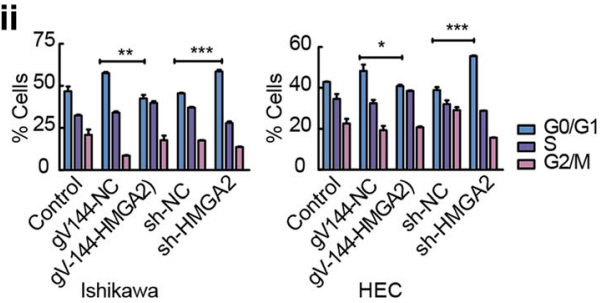

Fig. 2 HMGA2 plays an oncogenic role in Ishikawa and HEC-1A cell lines. a and $\mathbf{b}$ CCK-8 and EdU assays were used to detect the effect of HMGA2 expression on the proliferation of Ishikawa and HEC-1A cells. $\mathbf{c}$ Effects of different levels of HMGA2 on the migration of Ishikawa and HEC-1A cells were evaluated using a wound healing assay. $\mathbf{d}$ Transwell assays were used to detect cellular invasion. Representative images and accompanying statistical plots are presented. e The effects of different levels of HMGA2 on apoptosis were verified via flow cytometry. $\mathbf{f}$ Effect of HMGA2 on cell cycle distribution in Ishikawa and HEC-1A cell lines. Data are presented as the mean \pm SEM $\left(n=3\right.$ per group), ${ }^{*} P<0.05,{ }^{* *} P<0.01$, *** $P<0.0001$ vs. the NC group

positive rate of miR-367-3p expression in endometrial cancer was $47.5 \%$, which was lower than that in normal endometrial tissue (84.2\%) (Fig. 3e, Additional file 12: Table S9-i). Additionally, the correlations between the expression of miR-302a-5p/367-3p and HMGA2 protein in endometrial cancer patients were analysed. Spearman's rank correlation analysis found that the expression of miR-302a-5p/367-3p and the expression of HMGA2 in endometrial cancer were significantly negatively correlated miR-302a-5p: $\quad(r=-0.4316, \quad P<0.0001$, 


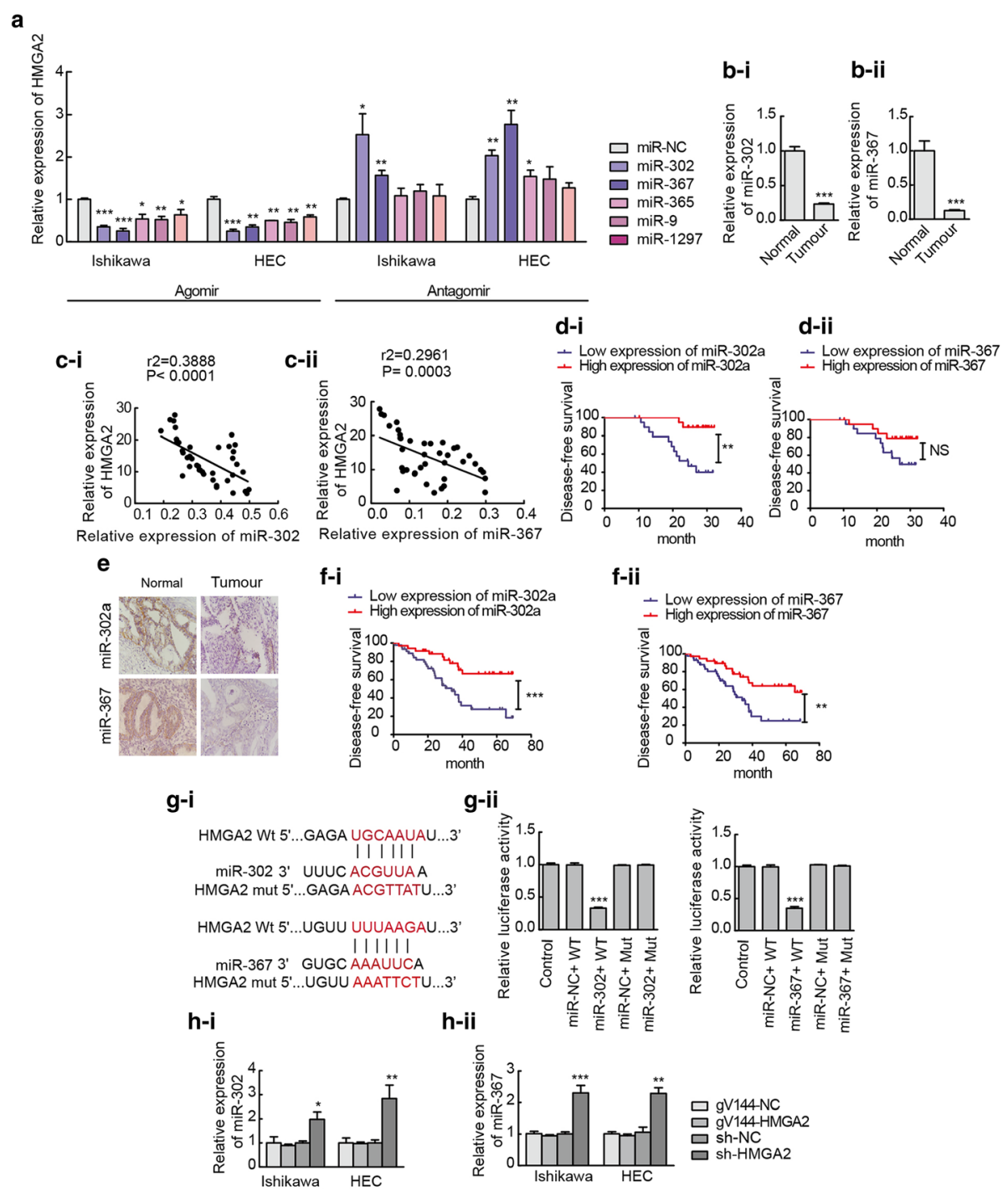

Fig. 3 HMGA2 is a target of miR-302a-5p and miR-367-3p. a Changes in miRNA expression and the expression of HMGA2 mRNA were detected via qRT-PCR in Ishikawa and HEC-1A cell lines. Data are presented as the mean \pm SEM ( $n=3$ per group). $\mathbf{b}$ miR-302a-5p/367-3p was downregulated in endometrial carcinoma tissues $(n=40)$ compared with normal tissues $(n=37)$. Data are presented as the mean \pm SEM. c A significant correlation between the levels of miR-302a-5p/367-3p and HMGA2 in endometrial carcinoma tissues $(n=40)$ was demonstrated using Pearson's correlation coefficient analysis. miR-302a-5p and HMGA2 $\left(r^{2}=0.3888, P<0.0001\right)$; miR-367-3p and HMGA2 $\left(r^{2}=0.2961, P=0.0003\right)$. d Disease-free survival curves for miR-302a-5p/367-3p in 40 endometrial carcinoma tissues $(n=40)$. NS, $P>0.05$. e and $\mathbf{f}$ Expression of miR-302a-5p/367-3p in 80 endometrial carcinoma tissues and disease-free survival curves. g Predicted miR-302a-5p/367-3p binding sites in the 3'-UTR of wild-type (HMGA2-3'-UTR-WT) and mutant (HMGA2-3'UTR-Mut) HMGA2 sequences. Luciferase reporter assay using HEK293T cells co-transfected with HMGA2-WT (or HMGA2-Mut) and the indicated miRNA. Data are presented as the mean \pm SEM ( $n=3$ per group). $\mathbf{h}$ Expression of miR-302a-5p/367-3p was detected using qRT-PCR after the expression of HMGA2 was altered in Ishikawa and HEC-1A cell lines. Data are presented as the mean \pm SEM. ( $n=3$ per group). ${ }^{*} P<0.05$, ${ }^{* *} P<0.01$, ${ }^{* * *} P<0.0001$ vs. the NC group

Additional file 11: Table S8-ii); miR-367-3p: $(r=-$ $0.2700, P=0.0154$, Additional file 12: Table S9-ii). We also analysed the association of the levels of miR-302a$5 \mathrm{p} / 367-3 \mathrm{p}$ with the clinicopathologic parameters (Additional file 11: Table S8-iii; Additional file 12: Table S9-iii) and the disease-free survival (Fig. 3f) of endometrial cancer patients. The results indicated that miR- 302a-5p/367-3p expression is associated with muscular invasion and metastasis in patients with endometrial cancer and that high expression of miR-302a-5p/367-3p is correlated with high survival rates in endometrial cancer. The dual-luciferase gene reporter assay demonstrated that miR-302a-5p/367-3p could bind to HMGA2 at the predicted binding sites (Fig. 3g). 
Interestingly, the expression of $\mathrm{miR}-302 \mathrm{a}-5 \mathrm{p} / 367-3 \mathrm{p}$ was increased after the HMGA2 gene was knocked down (Fig. 3h), Thus, HMGA2 is regulated by miR-302a$5 \mathrm{p} / 367-3 \mathrm{p}$ not only in a unidirectional manner but also in the form of a regulatory feedback loop. Hence, we hypothesize the existence of a regulatory feedback mechanism between miR-302a-5p/miR-367-3p and HMGA2.

\section{Overexpression of $\mathrm{miR}-302 \mathrm{a}-5 \mathrm{p} / 367-3 \mathrm{p}$ inhibits cell proliferation, migration, and invasion and promotes apoptosis and cell cycle arrest}

To explore the possible biological significance of miR$302 a-5 p / 367-3 p$ in endometrial cancer, Ishikawa and HEC-1A cells were transfected with agomir-302a-5p/ $367-3 p$ or antagomir-302a-5p/367-3p, and changes in proliferation, invasion, apoptosis and cell cycle distribution were detected. Cell proliferation was assessed using CCK-8 and EdU assays. Transfection of cells with agomir-302a-5p/367-3p decreased cell proliferation compared with that of transfection with miR-NC, but cell proliferation was promoted after transfection of cells with antagomir-302a-5p/367-3p (Fig. 4a and b). Wound healing and transwell assays were used to evaluate cell migration and invasion, respectively. The migratory and invasion abilities of Ishikawa and HEC-1A cells were reduced in response to the overexpression of miR-302a-5p/ 367-3p (Fig. 4c and d). Flow cytometry was used to detect the rate of apoptosis in these cells. The overexpression of miR-302a-5p/367-3p promoted apoptosis, whereas the knockdown of miR-302a-5p/367-3p inhibited apoptosis (Fig. 4e). Flow cytometry was used to analyse cell cycle distribution. Cells transfected with agomir-302a-5p/367$3 p$ were arrested at the G0/G1 phase (Fig. $4 f$ ).

\section{HMGA2 is a functional target of miR-302a-5p/367-3p in the regulation of EMT-associated proteins in Ishikawa and HEC- 1 A cell lines}

To further study the mechanism by which miR-302a-5p/ 367-3p and HMGA2 regulate cell migration and invasion, we examined the levels of EMT-associated proteins in Ishikawa and HEC-1A cells. The effects of HMGA2, miR302a-5p and miR-367-3p on EMT-related proteins were detected using western blotting. Knockdown of HMGA2 expression or overexpression of miR-302a-5p/367-3p decreased the expression of EMT-related proteins including Snail, Slug, N-cadherin, MMP-2, and MMP-9 and increased the expression of E-cadherin (Fig. 5a and b). In addition, the concentrations of MMP-2 and MMP-9 in the supernatant of Ishikawa and HEC-1A cell cultures were detected by Enzyme-linked immunosorbent assay (ELISA). The results showed that the knockdown of HMGA2 expression or the overexpression of miR-302a-5p/367-3p decreased the concentration of the MMP-2 and MMP-9 proteins in the cell culture medium, which suggests that interference in HMGA2 expression or the high expression of miR-302a-5p/367-3p inhibits Ishikawa and HEC-1A cell secretion of MMP-2 and MMP-9 proteins (Fig. $5 c$ and d). Therefore, overexpression of miR-302a-5p/367-3p or knockdown of HMGA2 reversed EMT in endometrial cancer cells. In other words, HMGA2 is a functional target of miR-302a-5p/367-3p in the regulation of EMTassociated proteins in Ishikawa and HEC-1A cell lines.

\section{miR-302a-5p/367-3p, HMGA2 and RUNX1 form a regulatory axis in endometrial carcinoma cells}

The results of qRT-PCR and the TCGA dataset analysis showed that the expression level of RUNX1 was higher in endometrial carcinoma tissues than that in normal endometrial tissues (Additional file 13: Figure S4a and b). Moreover, the expression of RUNX1 was positively correlated with the expression of HMGA2 in endometrial carcinoma tissue (Additional file 13: Figure $\mathrm{S} 4 \mathrm{c}$ and $\mathrm{d}$ ). To further study the mechanism by which RUNX1 regulate cell migration and invasion, we examined the expression of MMP-2 and MMP-9 in Ishikawa and HEC-1A cells. The results showed that overexpressed of RUNX1 increased the expression of MMP-2 and MMP-9 at the mRNA and protein levels (Additional file 13: Figure $\mathrm{S} 4 \mathrm{e}$ and $\mathrm{f}$ ). In addition, the results of ELISA showed that the overexpressed of RUNX1 increased the concentration of the MMP-2 and MMP-9 proteins in the cell culture medium (Additional file 13: Figure S4 g). Co-immunoprecipitation assays were performed in Ishikawa and HEC-1A cells. HMGA2 was found to be bound to RUNX1 (Additional file 13: Figure S4 h). To verify whether RUNX1 is involved in the regulation of HMGA2, HMGA2 was overexpressed or knocked down in Ishikawa and HEC-1A cells, and qRT-PCR and western blot analyses were performed to detect the mRNA and protein expression levels of RUNX1. Overexpression of HMGA2 increased the expression of RUNX1 at the mRNA and protein levels (Fig. 5e and f). We then investigated whether miR-302a5p/367-3p modulates RUNX1 expression through regulation of HMGA2. First, we screened the full RUNX1 mRNA sequence from TargetScan bioinformatics databases and identified no miR-302a-5p/367-3p binding sites. Then, Ishikawa and HEC-1A cells were co-transfected with overexpression constructs of miR-302a-5p/367-3p and HMGA2. Notably, rescue experiments demonstrated that miR-302a-5p/367-3p-dependent downregulation of RUNX1 protein was largely negated by overexpression of HMGA2 (Fig. 5g).

\section{miR-302a-5p/367-3p controls the HMGA2-mediated suppression of the malignant behaviour of endometrial carcinoma cells}

To verify whether miR-302a-5p/367-3p controls the HMGA2-mediated regulation of the malignant behaviour 

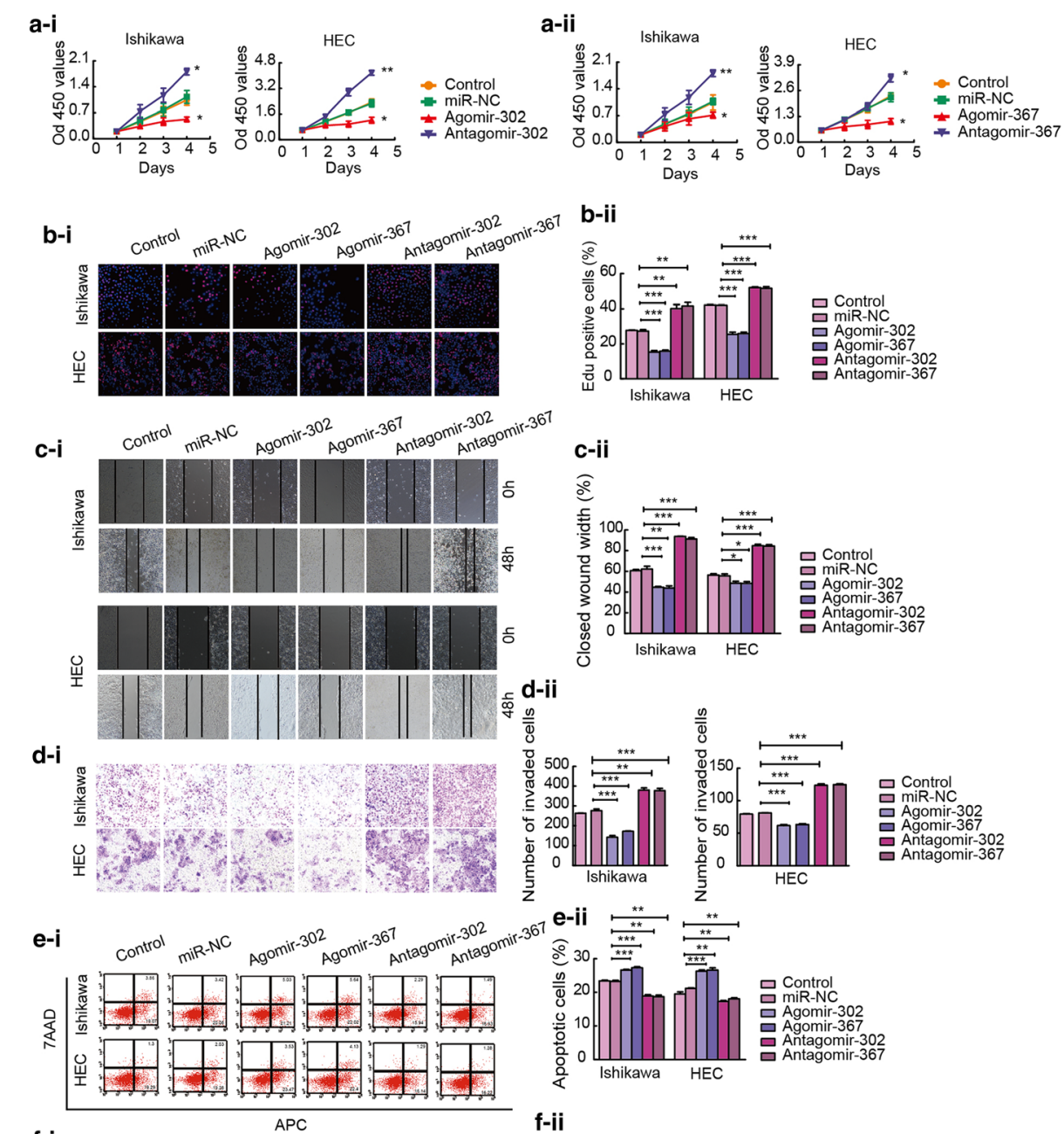

f-i
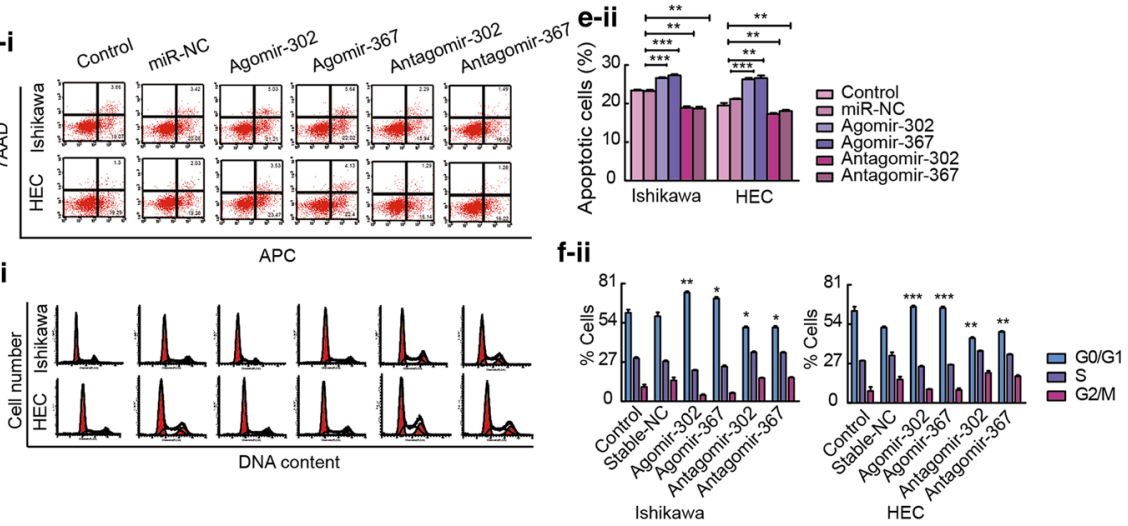

Fig. 4 miR-302a-5p/367-3p inhibits malignant biological behaviour of Ishikawa and HEC-1A cell lines. $\mathbf{a}$ and $\mathbf{b}$ CCK-8 and EdU assays were used to evaluate effect of miR-302a-5p/367-3p on the proliferation Ishikawa and HEC-1A cell lines. $\mathbf{c}$ Representative images of the wound healing assay in Ishikawa and HEC-1A cell lines in response to changes in the expression of miR-302a-5p/367-3p. $\mathbf{d}$ Transwell assays were used to detect the effect of miR-302a-5p/367-3p expression on invasion in Ishikawa and HEC-1A cell lines. Representative images and accompanying statistical plots are presented. e Effects of miR-302a-5p/367-3p on apoptosis in Ishikawa and HEC-1A cell lines. $\mathbf{f}$ Effects of miR-302a-5p/367-3p on cell cycle distribution in Ishikawa and HEC-1A cell lines. Data are presented as the mean \pm SEM ( $n=3$ per group). ${ }^{*} P<0.05$, ${ }^{* *} P<0.01,{ }^{* * *} P<0.0001$ vs. the miR-NC group

of endometrial carcinoma cells, we investigated the exact contribution of the miR-302a-5p/367-3pHMGA2 axis on Ishikawa and HEC-1A cells. Ishikawa and HEC-1A cells were co-transfected with a miR-302a-5p/367-3p overexpressing plasmid and gv144-HMGA2. The cells were divided into the following groups: control vector + miR-NC, control vector + agomir-302a-5p/367-3p and gV144-HMGA2 + agomir302a-5p/367-3p. The efficiency of HMGA2 re- expression was determined by western blot (Additional file 14: Figure S5). When cells were cotransfected with gV144-HMGA2 and agomir-302a-5p/ 367-3p, HMGA2 attenuated the suppressive effect of miR-302a-5p/367-3p on cell proliferation, invasion, and migration and prevented apoptosis and cell cycle arrest in the G0/G1 phase. Therefore, the rescue experiments implied that HMGA2 is a functional target of miR302a-5p/367-3p and ectopic expression of HMGA2 
a-i

E-Cadherin

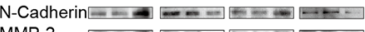

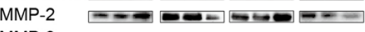

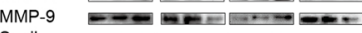

Snail

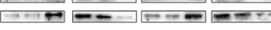
GAPDH
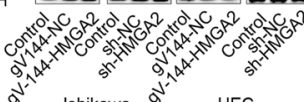

a-iii
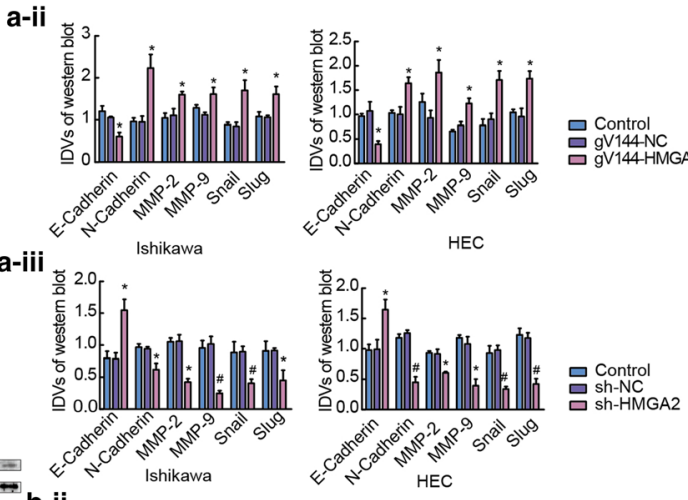

b-i

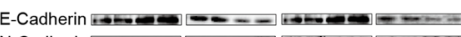
N-Cadherin

MMP-9

Snail

Slug
HMGA2

matand

-

APDH

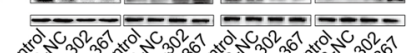

cis

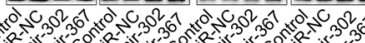

किक

Ishikawa

HEC

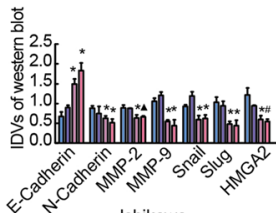

Ishikawa
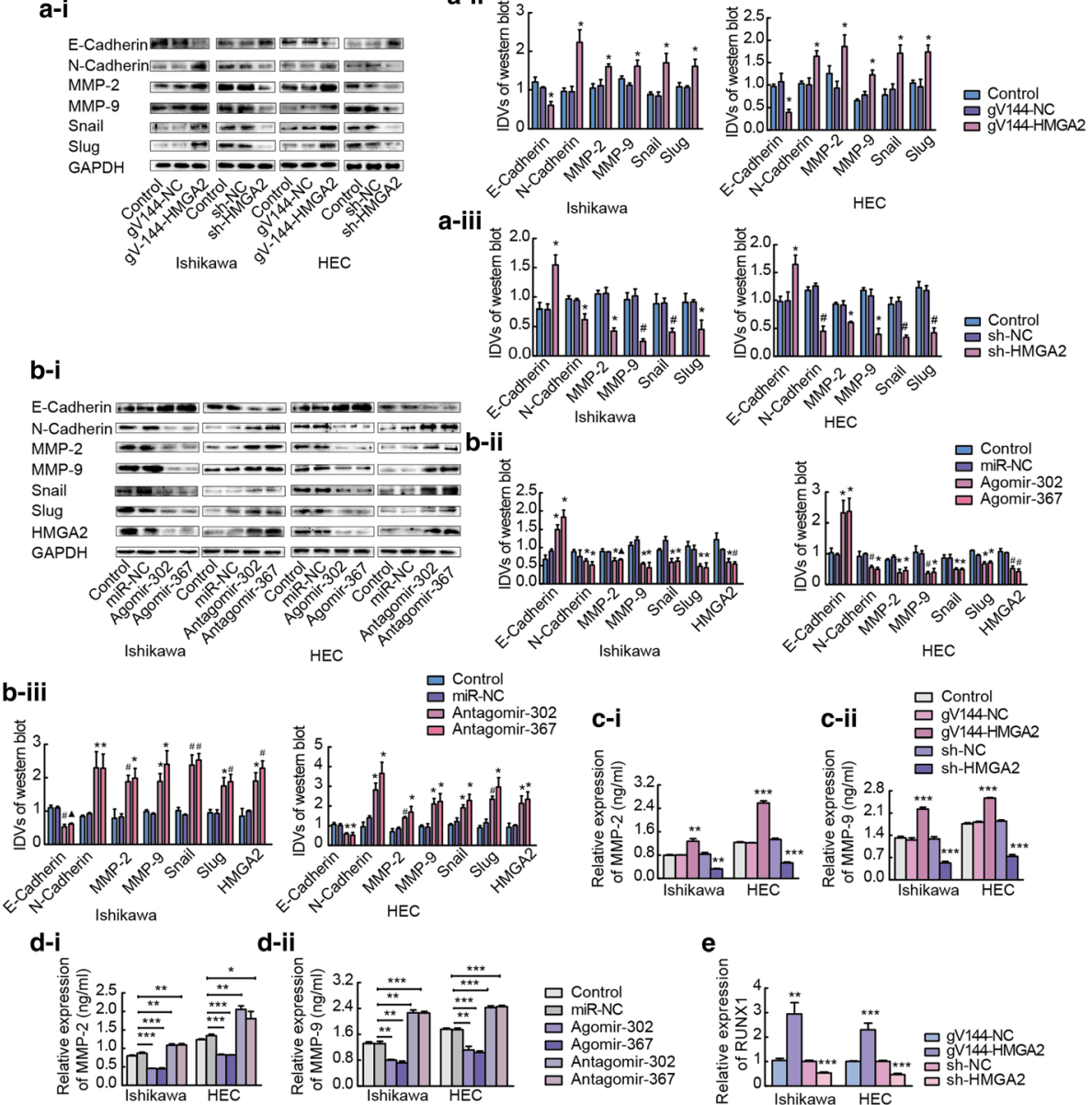

$\square$ Control

믐 Antagomir-302 $\quad$ C-i

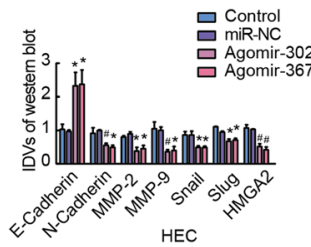

HEC
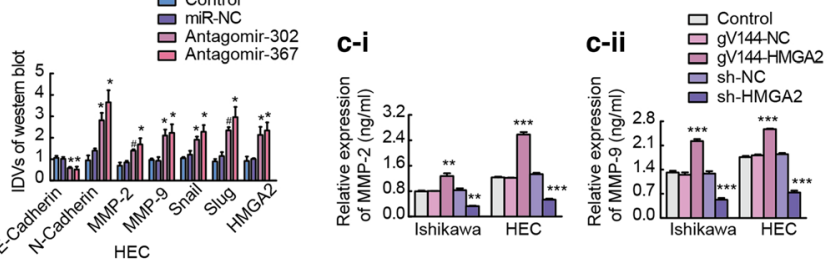

HEC

ii

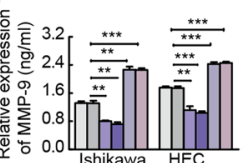

무 Control

Agomir-302

Agomir-367

Antagomir-302

口 Antagomir-367

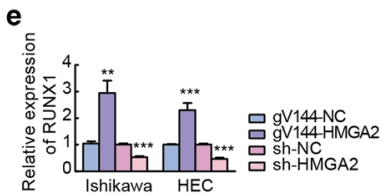

f-ii
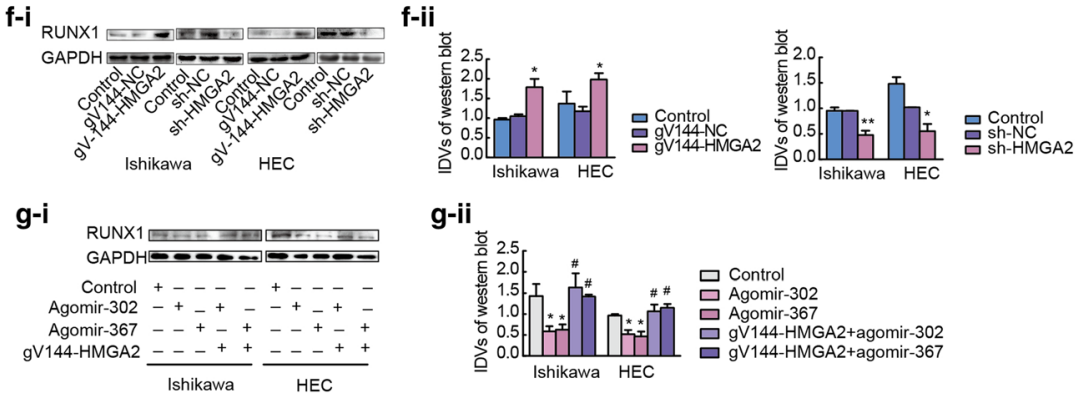

g-ii

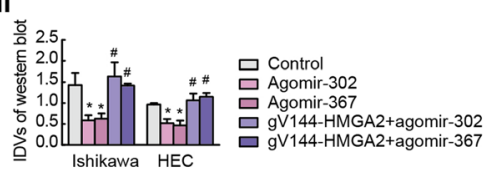

Fig. 5 HMGA2 is a functional target of miR-302a-5p/367-3p in regulating EMT. a Western blot analysis of the expression of EMT-related proteins including E-cadherin, N-cadherin, MMP-2, MMP-9, Snail and Slug in response to HMGA2 in Ishikawa and HEC-1A cell lines. Relative expression of $\mathrm{GAPDH}$ as an endogenous control. Data are presented as the mean $\pm \mathrm{SEM}$, ( $n=3$ per group). $\mathbf{b}$ Western blot analysis of the expression of EMT-related protein including E-cadherin, N-cadherin, MMP-2, MMP-9, Snail and Slug in response to miR-302a-5p/367-3p. Data are presented as the mean \pm SEM. ( $n=3$ per group), ${ }^{*} P<0.05, \# P<0.01, \boldsymbol{\Delta} P<0.0001$. $\mathbf{c}$ and $\mathbf{d}$ Concentrations of MMP-2 and MMP-9 proteins in the supernatant of Ishikawa and HEC-1A cell cultures were detected by ELISA ${ }^{*} P<0.05,{ }^{* *} P<0.01$, ${ }^{* *} P<0.0001$, ( $\mathrm{n}=3$ per group). e and $\mathbf{f}$ qRT-PCR and western blotting of HMGA2regulated RUNX1 expression in Ishikawa and HEC-1A cell lines. Data are presented as the mean \pm SEM. ${ }^{*} P<0.05$, ${ }^{* *} P<0.01$, ${ }^{* * *} P<0.0001$ vs. the NC group, ( $n=3$ per group). $\mathbf{g}$ Western blot analysis of RUNX1 protein levels in Ishikawa and HEC-1A cell. Data are presented as the mean \pm SEM. ${ }^{*} P<0.05,{ }^{* *} P<0.01,{ }^{* *} P<0.0001$ vs. the NC group, $(n=3$ per group) 
can reverse the anti-tumour effect of miR-302a-5p/ 367-3p (Fig. 6a-f).

\section{Overexpression of miR-302a-5p/367-3p combined with knockdown of HMGA2 significantly inhibits tumour growth in vivo}

To determine the function of HMGA2 and miR-302a$5 \mathrm{p} / 367-3 \mathrm{p}$ in vivo, we analysed the effects of miR-302a5p/367-3p overexpression or HMGA2 knockdown and their combinatorial effect on endometrial tumour xenografts in nude mice. Ishikawa and HEC-1A cells were co-transfected with a miR-302a-5p/367-3p-overexpressing plasmid and sh-HMGA2. The cells were divided into the following groups: control, miR-NC, agomir-302a-5p/ 367-3p, sh-NC, sh-HMGA2, and sh-HMGA2 + agomir302a-5p/367-3p. The expression of miR-302a-5p/367-3p was determined by qRT-PCR, and the expression of HMGA2 was determined by qRT-PCR and western blotting. The results showed that the expression of HMGA2 was the lowest in the sh-HMGA2 + agomir-302a-5p/ 367-3p group and the expression of miR-302a-5p/367$3 p$ was the highest in the Ishikawa and HEC-1A cells (Additional file 15: Figure S6). In vivo, the volume and weight of tumours were lower in the sh-HMGA2 group than in the sh-NC group. Additionally, the tumours in the agomir-302a-5p/367-3p group were smaller than those in the miR-NC group. In particular, tumours were the smallest in the group co-transfected with shHMGA2 with agomir-302a-5p/367-3p (Fig. 7a-c). According to the immunohistochemical assessment, relative to the agomir-302a-5p/367-3p and sh-HMGA2 groups, the expression of HMGA2 and Ki-67 was the lowest in the co-transfected group. In addition, the expression of EMT-related proteins including MMP-2, MMP-9, Snail, Slug, N-cadherin, and Ki-67 was lowest in the co-transfected group, whereas the expression of Ecadherin was the highest (Additional file 15: Figure S6).

\section{Discussion}

Our study demonstrated that high expression of HMGA2 correlates with poor clinical outcomes in endometrial cancer and that its expression is significantly correlated with clinical stage, tumour grade, and metastasis. Overexpression of HMGA2 promoted proliferation, migration, and invasion and inhibited apoptosis and cell cycle arrest in endometrial cancer cells, indicating that HMGA2 plays an oncogenic role in endometrial carcinoma. The results of this study provide compelling evidence on the function and role of HMGA2 in endometrial cancer.

miRNAs are involved in physiological processes such as cell differentiation, apoptosis, proliferation, embryonic development, and stem cell renewal. miRNAs are used as potential biomarkers in the detection and prognosis of cancer, with broad clinical applications [20]. Most
miRNAs hybridize to complementary bases on the 3'untranslated region (3'-UTR) of target mRNAs, leading to the negative regulation of target gene expression via translational inhibition or mRNA degradation [21]. Accumulating evidence has confirmed the abnormal expression of miRNAs in endometrial cancer [22]. miR302a-5p/367-3p, predicted by bioinformatics software (TargetScan and miRanda), can bind to the 3'-UTR of HMGA2 as demonstrated by the dual-luciferase reporter assay. We demonstrated that the expression level of HMGA2 was decreased after overexpression of miR302a-5p/367-3p in endometrial carcinoma cell lines. Additionally, the expression of miR-302a-5p/367-3p was detected after artificially altering the levels of HMGA2. On the one hand, miR-302a-5p/367-3p was found to regulate HMGA2 in a unidirectional manner; on the other hand, HMGA2 was found to regulate miR-302a$5 p / 367-3 p$ expression. Therefore, we concluded that there is a regulatory feedback loop between miR-302a5p/367-3p and HMGA2. The concept of feedback regulation of miRNAs by their target genes is widely accepted $[23,24]$. In previous studies, the miR-302 family was shown to exert anti-tumour effects in several cancers, including liver, cervical, ovarian and gastric cancers [25-28]. However, the function of miR-302-5p/367-3p in endometrial carcinoma cell lines has rarely been studied. In this study, we demonstrated that the expression of miR-302-5p/367-3p is low in endometrial carcinoma tissues and that miR-302a-5p/367-3p expression is associated with muscular invasion and metastasis. We also demonstrated that low expression of miR-302a-5p/367-3p is correlated with poor outcomes in endometrial cancer. In endometrial cancer cell lines, high levels of miR-302-5p/367-3p can inhibit malignant behaviours of cells.

Metastasis is one of the important reasons behind the high mortality rate among cancer patients. Therefore, understanding the mechanism of invasion and metastasis in endometrial carcinoma may provide the basis for molecular therapy to inhibit progression of endometrial cancer and enable the discovery of novel ways to treat and prevent cancer metastasis. EMT is characterized by a decrease in the expression of cellular adhesion molecules such as E-cadherin and an increase in the expression of intercellular proteins such as $\mathrm{N}$-cadherin, resulting in a mesenchymal cell morphology. Alterations in the expression of these proteins reduce cell-cell adhesion, leading to the proliferation of cancer cells and EMT, which is a key process in cancer metastasis $[29,30]$. Transcriptional regulators of the SNAIL family, including Snail and Slug, act as key transcription factors to regulate EMT by directly binding to a specific E-box on the Ecadherin promoter. The expression of Snail and Slug is an early event and plays a central role in EMT [31]. The 
a
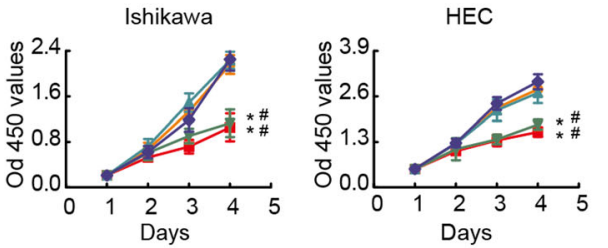

- Control+miR-NC

- Control+agomir-302

- gV144-HMGA2+agomir-302

- Control+agomir-367

Days

- gV144-HMGA2+agomir-367

b-i

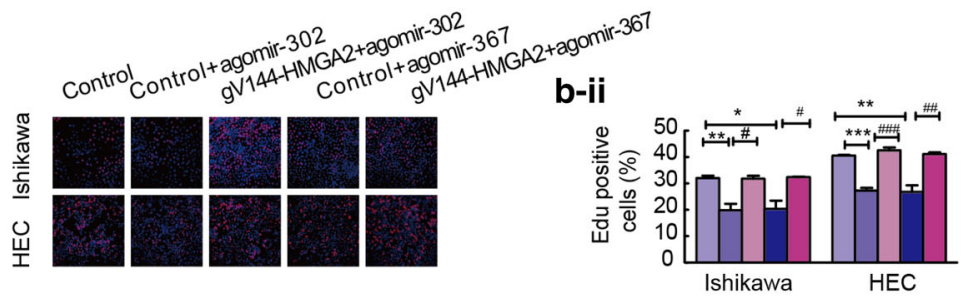

c-i

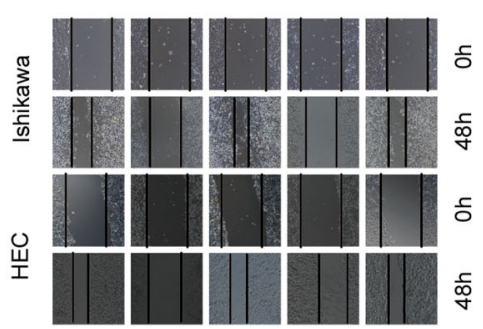

d-i
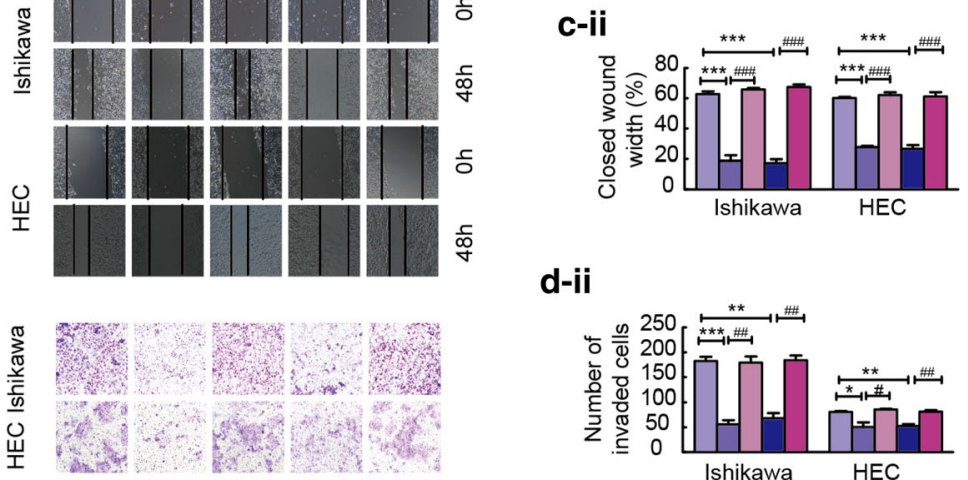

\section{d-ii}

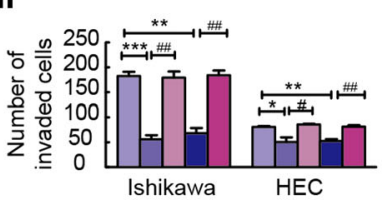

e-i

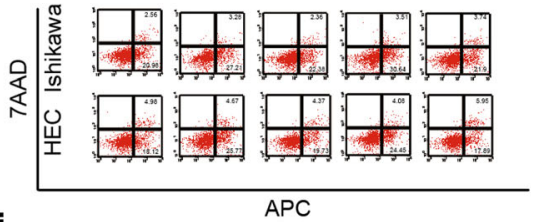

\section{e-ii}
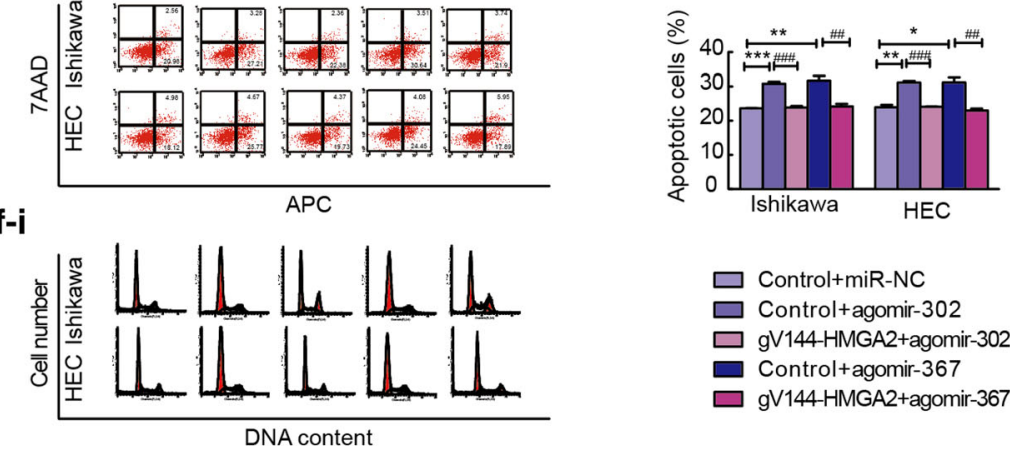

$\square$ Control+miR-NC

$\square$ Control+agomir-302

$\square$ gV144-HMGA2+agomir-302

$\square$ Control+agomir-367

$\square$ gV144-HMGA2+agomir-367

\section{f-ii}
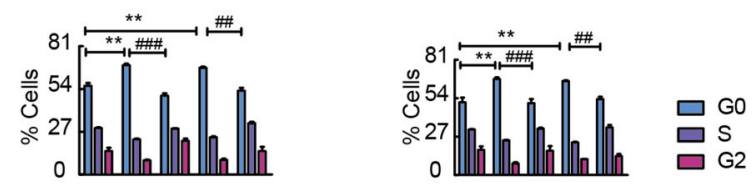

Control ++-+

miR-NC

Agomir-302 - + + -

Agomir-367 - - ++

gV144-HMGA2

Ishikawa

Control ++-+

miR-NC + - - -

Agomir-302 - + + -

Agomir-367 - -++

gV144-HMGA2 - +++

HEC

Fig. 6 (See legend on next page.) 
(See figure on previous page.)

Fig. 6 miR-302a-5p/367-3p suppresses the malignant behaviour of endometrial carcinoma cells by targeting HMGA2. a and b CCK-8 and EdU assays were used to detect proliferation in Ishikawa and HEC-1A cell lines after co-transfection with miR-302a-5p/367-3p and HMGA2-expression constructs. c A wound healing assay was performed to investigate the migratory ability of the cells. $\mathbf{d}$ Quantification of cell invasion in the groups with different expression levels of miR-302a-5p/367-3p and HMGA2. Representative images and statistical plots are presented. e Flow cytometric analysis of apoptosis in Ishikawa and HEC-1A cells expressing various levels of miR-302a-5p/367-3p and HMGA2. f Representative flow diagrams and graphs showing cell cycle distribution in the groups with different levels of miR-302a-5p/367-3p and HMGA2. Data are presented as the mean \pm SEM, $(n=3) .{ }^{*} P<0.05,{ }^{* *} P<0.01$ vs. control vector + miR-NC, $\# P<0.05$, \#\# $P<0.01$, \#\#\# $P<0.0001$ vs. control vector + agomir-302a-5p/367-3p

induction of EMT by MMPs has been well characterized in a variety of cancer cells [32], and it involves transmembrane protein regulation, including the release of the extracellular domain of E-cadherin and a reduction in adhesion. MMP-2 and MMP-9 have been found to be associated with invasive endometrial cancers [33]. HMGA2 is widely considered a driving factor of EMT in tumours; additionally, it is known that miRNA-mediated regulation of target genes can lead to reversal of EMT [34, 35]. In this study, the overexpression of miR-302a-5p/367-3p or knockdown of HMGA2 in endometrial cancer cells decreased the expression of EMT-related proteins, such as MMP-2, MMP-9, Snail, Slug and N-cadherin, while increasing the expression of E-cadherin. Thus, we confirmed that in endometrial cancer cells, HMGA2 is a functional target of miR-302a-5p/367-3p in the regulation of EMTassociated proteins. HMGA2, as an important regulatory factor for cancer cell metastasis, can directly affect the expression of EMT-related transcription factors, including Snail and Slug during the EMT process [36]. In addition, HMGA2 can regulate other genes and influence the processes of metastasis. Researchers have analysed the differences in gene expression profiles of tumour and non-tumour endometrial tissues and demonstrated the overexpression of RUNX1 in invasive endometrioid carcinoma tissues [37, 38]. A study by Planagumà et al. demonstrated the association of MMP-2 and MMP-9 with RUNX1 [39]. An orthotopic mouse model of endometrial cancer demonstrated that RUNX1 promotes distant metastasis [40]. Our study demonstrated that overexpressed of RUNX1 increased the expression of MMP-2 and MMP-9 in Ishikawa and HEC-1A cells. The results indicated that RUNX1 promotes distant metastasis in endometrial carcinoma at least partially dependent on the regulation of MMP-2 and MMP-9 expression. HMGA2 can regulate the transcription of several genes by enhancing or inhibiting transcription factor recruitment and affecting the expression of genes via protein-DNA or protein-protein interactions [41, 42]. We retrieved and analysed the data from the TCGA dataset to determine the relationship between the protein levels of HMGA2 and RUNX1 in endometrial carcinoma, and the results indicated that the expression of RUNX1 was positively correlated with the expression of HMGA2. Furthermore, a co-immunoprecipitation assay demonstrated that
HMGA2 was bound to RUNX1 protein. In addition, overexpression of HMGA2 increased the expression of RUNX1 at the mRNA and protein levels, whereas knockdown of HMGA2 had the opposite effect. Since HMGA2 is an important gene expression modulator in cancer, we proposed that, through regulation of HMGA2, miR-302a$5 p / 367-3 p$ also indirectly influences RUNX1 protein expression. Then, we confirmed that HMGA2 functions as a bridge to link miR-302a-5p/367-3p and RUNX1. Therefore, miR-302a-5p/367-3p likely regulates extracellular matrix remodelling during invasion and distant metastasis in endometrial cancer by regulating the miR-302a-5p/3673p-HMGA2 axis.

We further investigated the effect of the binding of miR-302a-5p/367-3p to HMGA2 on the malignant behaviour of endometrial carcinoma in vitro and found that the overexpression of HMGA2 attenuated the suppressive effect of miR-302a-5p/367-3p on the malignant behaviour of endometrial cancer cells. This result indicated that miR-302a-5p/367-3p regulates the malignant behaviour of endometrial carcinoma cells and is at least partially dependent on the regulation of HMGA2. From the assay used to determine the function of HMGA2 and miR-302a-5p/367-3p in vivo, we found that the overexpression of miR-302a-5p/367-3p combined with the knockdown of HMGA2 effectively reduced the volume and weight of transplanted tumours in nude mice. We further observed that the expression of HMGA2 was lowest in cell lines and nude mouse tissues, while the expression of miR-302a-5p/367-3p was highest in the shHMGA2 + agomir-302a-5p/367-3p group in cell lines. These results suggest that sh-HMGA2 combined with agomir-302a-5p/367-3p can interfere with HMGA2 expression and the expression of EMT-related proteins including MMP-2, MMP-9, Snail, Slug, N-cadherin to a maximum degree. In addition, knockdown of HMGA2 expression, increased the expression of miR-302a-5p/ 367-3p. Besides HMGA2, miR-302a or miR-367-3p may target multiple genes and pathways to inhibit tumour growth and metastasis [43, 44]. This indicates that the combination of agomirs and target genes may be more effective in the inhibition of tumour growth in vitro. It has been reported that in glioblastoma, agomir-101 combined with sh-KLF6 can reduce the size of tumours in nude mice more effectively than transfection of either 


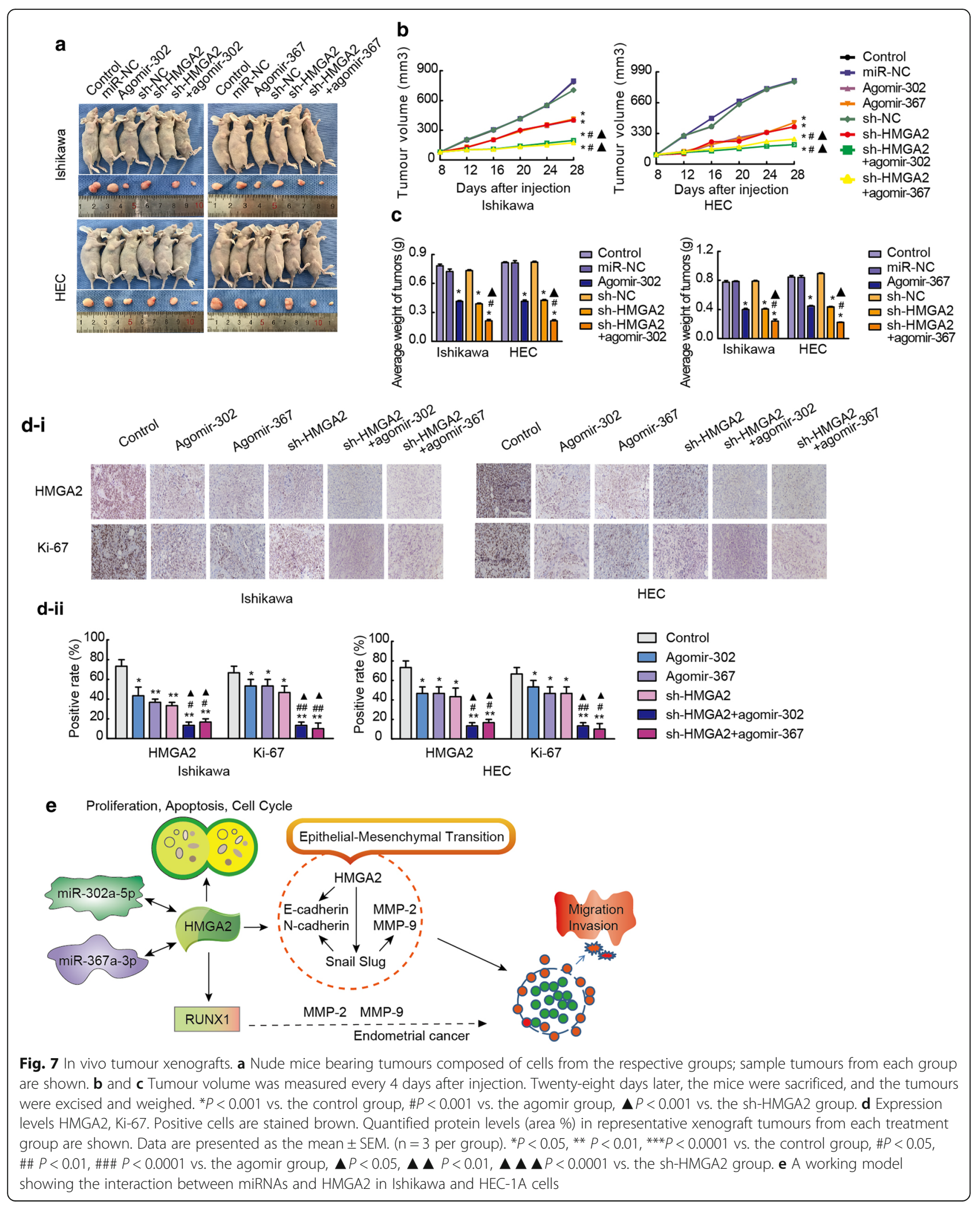


agomir-101 or sh-KLF6 alone and that the survival rate of nude mice is highest in the co-transfection group [45].

\section{Conclusions}

In summary, this study demonstrated that miR-302a-5p/ 367-3p mediated the ability of HMGA2 to regulate the malignant behaviour of endometrial carcinoma cells. A working model is shown in Fig. 7e. In the future, potential molecular therapies targeting the miRNA/HMGA2 axis should be explored as novel strategies to treat endometrial cancer.

\section{Additional files}

Additional file 1: Table $\mathbf{S 1}$. The shRNA clone and the agomir and antagomir sequences. (DOCX $17 \mathrm{~kb}$ )

Additional file 2: Table S2. Primer sequences for qRT-PCR. (DOCX $14 \mathrm{~kb}$ ) Additional file 3: Table S3. Primary antibodies used for the detection of protein expression. (DOCX $14 \mathrm{~kb}$ )

Additional file 4: Table S4. S4-i: The expression of HMGA2 protein in normal endometrial tissue $(n=19)$ and in endometrial carcinoma tissue $(n=80)$; S4-ii: Association between HMGA2 protein expression and the clinicopathologic characteristics of endometrial cancer patients. (DOCX $16 \mathrm{~kb}$ )

Additional file 5: Table S5. Association between HMGA2 mRNA expression and the clinicopathologic characteristics of endometrial cancer patients $(n=40)$. (DOCX $16 \mathrm{~kb})$

Additional file 6: Figure S1. a The baseline expression of HMGA2 in four endometrial cancer cell lines by qRT-PCR. $\mathbf{b}$ and $\mathbf{c}$ QRT-PCR and western blot analyses were used to determine the transfection efficiency of HMGA2. Data are presented as the mean \pm SEM ( $n=3$ per group). ${ }^{*} P<0.05$, ** $P<0.01$, *** $P<0.0001$. (PDF $507 \mathrm{~kb}$ )

Additional file 7: Figure S2. We employed TargetScan and miRanda bioinformatics databases to predict miRNAs that can bind to HMGA2. In total, 25 miRNAs were screened via co-transfection with wild-type dualluciferase vector-mediated HMGA2 constructs to identify the most suitable miRNAs. Data are presented as the mean \pm SEM ( $n=3$ per group). ${ }^{*} P<0.05$, ** $P<0.01$, *** $P<0.0001$. (PDF $800 \mathrm{~kb}$ )

Additional file 8: Figure S3. GRT-PCR was used to determine the transfection efficiency of the miRNAs. Data are presented as the mean \pm SEM ( $n=3$ per group). ${ }^{*} P<0.05,{ }^{* *} P<0.01,{ }^{* * *} P<0.0001$. (PDF $426 \mathrm{~kb}$ )

Additional file 9: Table S6. Association between miR-302a-5p expression and the clinicopathologic characteristics of endometrial cancer patients $(n=40)$. (DOCX $15 \mathrm{~kb})$

Additional file 10: Table S7. Association between miR-367-3p expression and the clinicopathologic characteristics of endometrial cancer patients $(n=40)$. (DOCX $15 \mathrm{~kb})$

Additional file 11: Table S8. S8-i: The expression of miR-302a-5p in normal endometrial tissue $(n=19)$ and in endometrial carcinoma tissue $(n=80)$; S8-ii: Spearman's rank correlation analysis of the correlations between expression of miR-302a-5p and HMGA2 protein in endometrial cancer patients $(n=80)$. S8-iii: Association between miR-302a-5p expression and the clinicopathologic characteristics of endometrial cancer patients $(n=80)$. (DOCX $17 \mathrm{~kb})$

Additional file 12: Table S9. S9-i: The expression of miR-367-3p in normal endometrial tissue $(n=19)$ and in endometrial carcinoma tissue $(n=80)$; S9-ii: Spearman's rank correlation analysis of the correlations between the expression of miR-367-3p and HMGA2 protein in endometrial cancer patients ( $n=80$ ). S9-iii: Association between miR-367-3p expression and the clinicopathologic characteristics of endometrial cancer patients $(n=80)$. (DOCX $18 \mathrm{~kb})$

Additional file 13: Figure S4. $\mathbf{a}$ and $\mathbf{b}$ The expression level of RUNX1 in endometrial carcinoma tissues. $\mathbf{c}$ and $\mathbf{d}$ The expression of RUNX1 was positively correlated with the expression of HMGA2 in endometrial carcinoma tissue. e to $\mathbf{g}$ Overexpressed of RUNX1 increased the expression of MMP-2 and MMP-9 in Ishikawa and HEC-1A cells. $\mathbf{h}$ A co-immunoprecipitation assay was used to validate HMGA2 was bound to RUNX1 in Ishikawa and HEC-1A cell lines. Data are presented as the mean \pm SEM ( $n=3$ per group). ${ }^{*} P<0.05$, ${ }^{* *} P<0.01$, ${ }^{* * *} P<0.0001$. (PDF $1149 \mathrm{~kb}$ )

Additional file 14: Figure S5. The expression of HMGA2 was determined by western blot. Data are presented as the mean \pm SEM ( $n=3$ per group). ${ }^{*} P<0.05,{ }^{* *} P<0.01,{ }^{* * *} P<0.0001$. (PDF $476 \mathrm{~kb}$ )

Additional file 15: Figure S6. a The expression of miR-302a-5p/367-3p was determined by qRT-PCR in Ishikawa and HEC-1A cells. b HMGA2 expression was determined by QRT-PCR and western blotting in Ishikawa and HEC-1A cells. Data are presented as the mean \pm SEM $(n=3$ per group). c Expression of EMT-related proteins including MMP-2, MMP-9, Snail, Slug and $\mathrm{N}$-cadherin were detected via immunohistochemistry. ${ }^{*} P<0.05$, ${ }^{* *} P<0.01,{ }^{* * *} P<0.0001$ vs. the control group, $\# P<0.05$, \#\# $P<0.01$, \#\#\# $P<0.0001$ vs. the agomir group, $\boldsymbol{\Delta} P<0.05$, $\boldsymbol{\Delta} \boldsymbol{\Delta} P<0.01$, $\boldsymbol{\Delta} \boldsymbol{\Delta} \boldsymbol{\Delta} P<0.0001$ vs. the sh-HMGA2 group. (PDF $6632 \mathrm{~kb}$ )

\section{Abbreviations}

3'-UTR: 3'-untranslated region; AUC: Area under the curve; ELISA: Enzymelinked immunosorbent assay; EMT: Epithelial-mesenchymal transition; FBS: Fetal bovine serum; HMGA2: High mobility group AT-hook 2; ISH: In situ hybridization; miRNAs: MicroRNAs; MMP-2: Matrix metalloproteinase-2; MMP-9: Matrix metalloproteinase-9; NC: Negative control; NS: Non-significance; PVDF: Polyvinylidene difluoride; qRT-PCR: Quantitative real-time PCR; ROC: Receiver operating characteristic; RUNX1: Runt related transcription factor 1; SDS-PAGE: Sodium dodecyl sulfate-polyacrylamide gel electrophoresis;

TCGA: The cancer genome atlas

\section{Acknowledgements}

We sincerely thank Yixue Xue for critical comments regarding experiments described in this article.

\section{Funding}

This work was supported by the National Natural Science Foundation of China (grant number 81272874, 81472438), the Department of Science and Technology of Liaoning Province (grant number 2013225079), Shenyang City Science and Technology Bureau (grant number F14-158-9-47), and the Outstanding Scientific Fund of Shengjing Hospital (grant number 201601). Funding for open access was provided by the National Natural Science Foundation of China (grant number 81272874, 81472438).

\section{Availablity of data and meterials}

The datasets and materials were available in current study.

\section{Authors' contributions}

$J M$ performed most of the experiments and contributed to the writing of the manuscript. JM, DL and XXM conceived of the study, participated in its design and coordination and helped draft the manuscript. JM, FFK, DY and HY performed the cell culture experiments. All authors read and approved the final manuscript.

\section{Ethics approval and consent to participate}

The study protocol was reviewed and approved by the Scientific Research and New Technology Ethical Committee of the Shengjing Hospital of China Medical University. Ethical number: 2014PS75K.

\section{Consent for publication}

We have obtained consents to publish this paper from all the participants of this study.

\section{Competing interests}

The authors declare that they have no competing interests.

\section{Publisher's Note}

Springer Nature remains neutral with regard to jurisdictional claims in published maps and institutional affiliations. 


\section{Received: 25 September 2017 Accepted: 23 January 2018}

\section{Published online: 01 February 2018}

\section{References}

1. Moore K, Brewer MA. Endometrial cancer: is this a new disease? Am Soc Clin Oncol Educ Book. 2017;37:435-42.

2. Slomovitz BM, Lu KH, Johnston T, Coleman RL, Munsell M, Broaddus RR, Walker C, Ramondetta LM, Burke TW, Gershenson DM, Wolf J. A phase 2 study of the oral mammalian target of rapamycin inhibitor, everolimus, in patients with recurrent endometrial carcinoma. Cancer. 2010;116:5415-9.

3. Kassem L, Abdel-Rahman O. Targeting mTOR pathway in gynecological malignancies: biological rationale and systematic review of published data. Crit Rev Oncol Hematol. 2016;108:1-12.

4. Yu Y, Hall T, Eathiraj S, Wick MJ, Schwartz B, Abbadessa G. In-vitro and in-vivo combined effect of ARQ 092, an AKT inhibitor, with ARQ 087, a FGFR inhibitor. Anti-Cancer Drugs. 2017;28:503-13.

5. Sgarra R, Zammitti S, Lo Sardo A, Maurizio E, Arnoldo L, Pegoraro S, Giancotti V, Manfioletti G. HMGA molecular network: from transcriptional regulation to chromatin remodeling. Biochim Biophys Acta. 1799;2010:37-47.

6. Pallante P, Sepe R, Puca F, Fusco A. High mobility group a proteins as tumor markers. Front Med (Lausanne). 2015;2:15.

7. Fusco A, Fedele M. Roles of HMGA proteins in cancer. Nat Rev Cancer. 2007; 7:899-910.

8. Mahajan A, Liu Z, Gellert L, Zou X, Yang G, Lee P, Yang X, Wei JJ. HMGA2: a biomarker significantly overexpressed in high-grade ovarian serous carcinoma. Mod Pathol. 2010;23:673-81.

9. Agostini A, Brunetti M, Davidson B, Trope CG, Heim S, Panagopos I, Micci F. Genomic imbalances are involved in miR-30c and let-7a deregulation in ovarian tumors: implications for HMGA2 expression. Oncotarget. 2017;8: 21554-60.

10. Mansoori B, Mohammadi A, Shirjang S, Baradaran B. HMGl-C suppressing induces P53/caspase9 axis to regulate apoptosis in breast adenocarcinoma cells. Cell Cycle. 2016;15:2585-92.

11. Rice SJ, Lai SC, Wood LW, Helsley KR, Runkle EA, Winslow MM, Mu D. MicroRNA-33a mediates the regulation of high mobility group AT-hook 2 gene (HMGA2) by thyroid transcription factor 1 (TTF-1/NKX2-1). J Biol Chem. 2013;288:16348-60.

12. Wei L, Liu X, Zhang W, Wei Y, Li Y, Zhang Q, Dong R, Kwon JS, Liu Z, Zheng $W$, et al. Overexpression and oncogenic function of HMGA2 in endometrial serous carcinogenesis. Am J Cancer Res. 2016;6:249-59.

13. Widodo, Djati MS, Rifa'i M. Role of MicroRNAs in carcinogenesis that potential for biomarker of endometrial cancer. Ann Med Surg. 2016;7:9-13.

14. Hebert C, Norris K, Scheper MA, Nikitakis N, Sauk JJ. High mobility group A2 is a target for miRNA-98 in head and neck squamous cell carcinoma. Mol Cancer. 2007:6:5

15. Santamaria PG, Moreno-Bueno G, Portillo F, Cano A. EMT: present and future in clinical oncology. Mol Oncol. 2017;17:718-38.

16. Ye X, Weinberg RA. Epithelial-Mesenchymal plasticity: a central regulator of cancer progression. Trends Cell Biol. 2015;25:675-86.

17. Lin Y, Liu AY, Fan C, Zheng H, Li Y, Zhang C, Wu S, Yu D, Huang Z, Liu F, Luo Q, Yang CJ, Ouyang G. MicroRNA-33b inhibits breast cancer metastasis by targeting HMGA2, SALL4 and Twist1. Sci Rep. 2015;5:9995.

18. Wang H, Jiang Z, Chen H, Wu X, Xiang J, Peng J. MicroRNA-495 inhibits gastric cancer cell migration and invasion possibly via targeting high mobility group AT-hook 2 (HMGA2). Med Sci Monit. 2017;23:640-8.

19. Chen KJ, Hou Y, Wang K, Li J, Xia Y, Yang XY, Lv G, Xing XL, Shen F. Reexpression of let-7g microRNA inhibits the proliferation and migration via K-Ras/HMGA2/snail axis in hepatocellular carcinoma. Biomed Res Int. 2014; 742417

20. Berindan-Neagoe I, Monroig Pdel C, Pasculli B, Calin GA. MicroRNAome genome: a treasure for cancer diagnosis and therapy. CA Cancer J Clin. 2014;64:311-36.

21. Ricci EP, Limous T, Soto-Rifo R, Rubilar PS, Decimo D, Ohlmann T. miRNA repression of translation in vitro takes place during 435 ribosomal scanning. Nucleic Acids Res. 2013;41:586-98.

22. Montagnana M, Benati M, Danese E, Giudici S, Perfranceschi M, Ruzzenenere O, Salvagno GL, Bassi A, Gelati M, Paviati E. Aberrant MicroRNA expression in patients with endometrial cancer. Int J Gynecol Cancer. 2017;27:459-66.

23. Wang X, Zhao Y, Qian H, Huang J, Cui F, Mao Z. The miR-101/RUNX1 feedback regulatory loop modulates chemo-sensitivity and invasion in human lung cancer. Int J Clin Exp Med. 2015:8:15030-42.
24. Wang S, Raghavachari S. Quantifying negative feedback regulation by micro-RNAs. Phys Biol. 2011:8:055002.

25. Wang L, Yao J, Shi X, Hu L, Li Z, Song T, Huang C. MicroRNA-302b suppresses cell proliferation by targeting EGFR in human hepatocellular carcinoma SMMC-7721 cells. BMC Cancer. 2013;13:448.

26. Cai N, Wang YD, Zheng PS. The microRNA-302-367 cluster suppresses the proliferation of cervical carcinoma cells through the novel target AKT1. RNA. 2013;19:85-95.

27. Guo T, Yu W, Lv S, Zhang C, Tian Y. MiR-302a inhibits the tumorigenicity of ovarian cancer cells by suppression of SDC1. Int J Clin Exp Pathol. 2015;8: 4869-80.

28. Khalili M, Sadeghzaden M, Ghorbanian K, Malekzaden R, Vasei M, Mowla SJ. Down-regulation of miR-302b, an ESC-specific microRNA, in gastric adenocarcinoma. Cell J. 2012;13:251-8.

29. Liu F, Gu LN, Shan BE, Geng CZ, Sang MX. Biomarkers for EMT and MET in breast cancer: an update. Oncol Lett. 2016;12:4869-76.

30. Nantajit D, Lin D, Li JJ. The network of epithelial-mesenchymal transition: potential new targets for tumor resistance. J Cancer Res Clin Oncol. 2015; 141:1697-713.

31. Gonzalez DM, Medici D. Signaling mechanisms of the epithelialmesenchymal transition. Sci Signal. 2014;7:re8.

32. Nistico P, Bissell MJ, Radisky DC. Epithelial-mesenchymal transition: general principles and pathological relevance with special emphasis on the role of matrix metalloproteinases. Cold Spring Harb Perspect Biol. 2013;4:a011908.

33. Divine LM, Nguyen MR, Meller E, Desai RA, Arif B, Rankin EB, Bligarn KH, Meyerson C, Hagemann IS, Massad M. AXL modulates extracellular matrix protein expression and is essential for invasion and metastasis in endometrial cancer. Oncotarget. 2016;7:77291-305.

34. Zhou W, Zou B, Liu L, Cui K, Gao J, Yuan S, Cong N. MicroRNA-98 acts as a tumor suppressor in hepatocellular carcinoma via targeting SALL4. Oncotarget. 2016;7:74059-73.

35. Zhao H, Kang X, Xia X, Wo L, Gu X, Hu Y, Xie X, Chang H, Lou L, Shen X. miR145 suppresses breast cancer cell migration by targeting FSCN-1 and inhibiting epithelial-mesenchymal transition. Am J Transl Res. 2016;8:3106-14.

36. Lamouille $\mathrm{S}, \mathrm{Xu}$ J, Derynck R. Molecular mechanisms of epithelialmesenchymal transition. Nat Rev Mol Cell Biol. 2014;15:178-96.

37. Planaguma J, Diaz-Fuertes M, Gil-Moreno A, Abal M, Monge M, Garcia A Baro T, Thomson TM, Xercavins J, Alameda F. A differential gene expression profile reveals overexpression of RUNX1/AML1 in invasive endometrioid carcinoma. Cancer Res. 2004;64:8846-53.

38. Planaguma J, Gonzalez M, Doll A, Monge M, Gil-Moreno A, Baro T, Garcia A, Xercavin J, Alameda F, Abal M. The up-regulation profiles of p21WAF1/CIP1 and RUNX1/AML1 correlate with myometrial infiltration in endometrioid endometrial carcinoma. Hum Pathol. 2006:37:1050-7.

39. Planaguma J, Liljestrom M, Alameda F, Butzom R, Virtanen I, Reventos J, Hukkanen M. Matrix metalloproteinase-2 and matrix metalloproteinase- 9 codistribute with transcription factors RUNX1/AML1 and ETV5/ERM at the invasive front of endometrial and ovarian carcinoma. Hum Pathol. 2011;42:57-67.

40. Doll A, Gonzalez M, Abal M, Llaurado M, Rigau M, Colas E, Monge M, Xercavins J, Capella G, Diaz B. An orthotopic endometrial cancer mouse model demonstrates a role for RUNX1 in distant metastasis. Int J Cancer. 2009;125:257-63.

41. Reeves R. Molecular biology of HMGA proteins: hubs of nuclear function. Gene. 2001;277:63-81.

42. Narita M, Narita M, Krizhanovsky V, Nunez S, Chicas A, Hearn SA, Myers MP, Lowe SW. A novel role for high-mobility group a proteins in cellular senescence and heterochromatin formation. Cell. 2006;126:503-14.

43. Qin C, Zha W, Fan R, Ding H, Xu Y, Wang C. MicroRNA302a inhibits cell proliferation and invasion, and induces cell apoptosis in hepatocellular carcinoma by directly targeting VEGFA. Mol Med Rep. 2017;16:6360-7.

44. Ding D, Zhang $Y$, Wen L, Fu J, Bai X, Fan Y, Lin Y, Dai H, Li Q, Zhang Y, An R. MiR-367 regulates cell proliferation and metastasis by targeting metastasisassociated protein 3 (MTA3) in clear-cell renal cell carcinoma. Oncotarget. 2017:8:63084-95.

45. Yao YL, Ma J, Wang P, Xue YX, Li Z, Zhao LN, Li ZQ, Feng TD, Liu YH. miR101 acts as a tumor suppressor by targeting Kruppel-like factor 6 in glioblastoma stem cells. CNS Neurosci Ther. 2015;21:40-51. 